\title{
A High-Performance Genetically Encoded Fluorescent Biosensor for Imaging Physiological Peroxynitrite
}

Zhijie Chen, ${ }^{1,3} 4$ Shen Zhang, ${ }^{2,4}$, Xinyu $\mathrm{Li}^{2}$, and Hui-wang $\mathrm{Ai}^{1,2,4 *}$

${ }^{1}$ Department of Chemistry, University of California, Riverside, Riverside, California 92521, USA.

2

Department of Molecular Physiology and Biological Physics, Department of Chemistry, Center

for Membrane and Cell Physiology, and the UVA Cancer Center, University of Virginia,

Charlottesville, Virginia 22908, USA.

3

3 Present Address: Institute for Quantitative Biosciences-QB3, University of California,

Berkeley, Berkeley, CA, 94720, USA.

4

These authors contributed equally

5

Lead Contact

*Correspondence: huiwang.ai@ virginia.edu (H.A.) 


\section{In Brief}

Chen et al. report pnGFP-Ultra, a high-performance fluorescent biosensor for minimally invasive and selective imaging of peroxynitrite production in live cells.

\section{Highlights}

- pnGFP-Ultra is a genetically encoded peroxynitrite biosensor with a 123-fold fluorescence turn-on response

- pnGFP-Ultra exhibits high selectivity toward peroxynitrite, with virtually no crossreaction with hydrogen peroxide

- An optimized plasmid-based system increases noncanonical amino acid incorporation in mammalian cells by $>10$ fold

- pnGFP-Ultra robustly detects peroxynitrite production in macrophages and primary glial cells 


\section{Summary}

Peroxynitrite is a highly reactive nitrogen species (RNS) that plays critical roles in signal transduction, stress response, and numerous human diseases. Advanced molecular tools that permit the selective, sensitive, and non-invasive detection of peroxynitrite is essential for understanding its pathophysiological functions. Here, we present pnGFP-Ultra, a high performance, reaction-based, genetically encodable biosensor for imaging peroxynitrite in live cells. pnGFP-Ultra features a $p$-boronophenylalanine-modified chromophore as the sensing moiety and exhibits a remarkable 123-fold fluorescence turn-on response towards peroxynitrite while displaying virtually no cross-reaction with other reactive oxygen/nitrogen species, including hydrogen peroxide. To facilitate the expression of pnGFP-Ultra in mammalian cells, we engineered a highly efficient noncanonical amino acid (ncAA) expression system that is broadly applicable to the mammalian expression of proteins containing various ncAAs. pnGFPUltra robustly detected peroxynitrite production during interferon $\gamma$ and lipopolysaccharideinduced immune responses in macrophages, and in amyloid $\beta$-activated primary glial cells. Thus, pnGFP-Ultra fills an important technical gap and represents an important new addition to the molecular toolbox in probing RNS biology.

\section{Keywords}

peroxynitrite $\bullet$ reactive nitrogen species $\bullet$ biosensor $\bullet$ fluorescent probes $\bullet$ genetic code expansion - noncanonical amino acids • live cell imaging • protein engineering • peroxynitrite production • fluorescence microscopy 


\section{Introduction}

Reactive oxygen species (ROS) has been studied intensively for decades, but our understanding of its cousin, reactive nitrogen species (RNS) lagged far behind. Peroxynitrite $\left(\mathrm{ONOO}^{-}\right)$is a RNS formed from a diffusion-controlled reaction between nitric oxide (NO•) and superoxide anion $\left(\mathrm{O}_{2}{ }^{-}{ }^{-}\right)$(Radi, 2013a), and has been implicated in numerous pathophysiological processes, including cardiac (Mungrue et al., 2002; Ronson et al., 1999), vascular (Förstermann and Münzel, 2006), circulatory (Szabó, 1996), inflammatory (Van Der Veen et al., 1997), diabetic (Zou et al., 2004), and neurodegenerative (Smith et al., 1997; Torreilles et al., 1999) diseases. Although no enzyme dedicated for direct peroxynitrite production has been identified, enzymes responsible for the biogenesis of the parental species of $\mathrm{ONOO}^{-}$are widely known: NO• (Beckman and Koppenol, 1996) and $\mathrm{O}_{2}{ }^{--}$(Fridovich, 1997) can be generated by nitric oxide synthetase (NOS) and NADPH oxidase (NOX) (Brewer et al., 2015), respectively (Figure 1A). In addition, the mitochondrial electron transport chain has been recognized as an important source of $\mathrm{O}_{2}^{--}$(Brand, 2016). These primary species present limited cellular toxicity but can react with each other and/or transition metals to produce highly reactive secondary species (e.g. hypochlorous acid, hydroxy radical, peroxynitrite) that could have deleterious consequences. For example, the direct toxicity of $\mathrm{NO} \bullet$ is modest but can be greatly enhanced by reacting with $\mathrm{O}_{2}{ }^{--}$ to produce $\mathrm{ONOO}^{-}$. As such, many nitrosative stresses historically attributed to NO• have been gradually reassigned to $\mathrm{ONOO}^{-}$(Pacher et al., 2007), a far more potent RNS capable of mediating radicals $\left(\bullet \mathrm{OH}, \mathrm{CO}_{3}{ }^{--}\right.$and $\left.\bullet \mathrm{NO}_{2}\right)$ generation, DNA damage, lipid peroxidation, protein nitration, and cell death (apoptosis or necrosis) (Szabó et al., 2007). In particular, $\mathrm{ONOO}^{-}$mediated post-translational protein tyrosine nitration plays important roles in cell signal transduction (Liaudet et al., 2009). In macrophages, $\mathrm{ONOO}^{-}$protects cells against invading pathogens by acting as a strong oxidant (Allen et al., 2012). Thus, like many reactive oxygen/nitrogen species (ROS/RNS), peroxynitrite displays a sophisticated signal/stress dichotomy that is inextricably linked to its complex biology (Pacher et al., 2007).

Elucidating the various roles $\mathrm{ONOO}^{-}$plays, whether in the context of normal cell signaling or pathogenesis, is pivotal for developing targeted therapeutics (Beckman, 2009; Szabó et al., 2007). Efforts to study $\mathrm{ONOO}^{-}$encounter similar hurdles to that of studying other ROS/RNS, such as a very short half-life, permeability through and reactions within membrane, multiplex 
reaction pathways, and signal/stress dichotomy (Ferrer-Sueta and Radi, 2009; Hardy et al., 2018). Traditionally, detection of $\mathrm{ONOO}^{-}$relied primarily on immunostaining of 3-nitrotyrosine footprints, but this method suffers from poor antibody specificity, a requisite for cell lysis, and interference from other nitration sources (Radi, 2013b). More recently, fluorescent probes for ONOO$^{-}$(Chen et al., 2013; Li et al., 2015; Lin et al., 2013; Peng and Yang, 2010; Peng et al., 2014; Sikora et al., 2009; Sun et al., 2014, 2015, 2009; Tian et al., 2011; Ueno et al., 2006; Xu et al., 2011; Yang et al., 2006; Yu et al., 2011, 2013; Zhang et al., 2012; Zielonka et al., 2010) have emerged as powerful tools for $\mathrm{ONOO}^{-}$detection, due to their high sensitivity, spatial-temporal resolution, and compatibility with well-established fluorescence microscopy platforms (Nadler and Schultz, 2013; Ueno and Nagano, 2011). Deployment of these tools in pathophysiological relevant settings such as in activated macrophage cells (Weber et al., 2020), in live smooth muscles from a mouse model of atherosclerosis (Peng et al., 2014), and in the cerebral vasculature of live mice ( $\mathrm{Li}$ et al., 2015), has revealed unprecedentedly rich information on peroxynitrite production and pathogenesis. Despite the progress, developing probes with both high reactivity and high selectivity for $\mathrm{ONOO}^{-}$remains a fundamental challenge (Chen et al., 2016a; Hardy et al., 2018). Of all the peroxynitrite probes reported thus far, none have shown absolute selectivity towards $\mathrm{ONOO}^{-}$over other ROS/RNS such as $\mathrm{H}_{2} \mathrm{O}_{2}, \bullet \mathrm{OH}$ and $\mathrm{ClO}^{-}$. For example, HKGreen-4, one of the most advanced probe for $\mathrm{ONOO}^{-}$, shows reactivity toward $\bullet \mathrm{OH}$ and $\mathrm{ClO}^{-}$at 1 eq probe concentration $(1 \mu \mathrm{M})$ (Peng et al., 2014); NP3, a probe with high $\mathrm{ONOO}^{-}$ sensitivity cross reacts (albeit mildly) with $\mathrm{H}_{2} \mathrm{O}_{2}$ and other species at 2 eq probe concentration $(10 \mu \mathrm{M})\left(\mathrm{Li}\right.$ et al., 2015); Probe 1-D-fructose shows substantial cross-reaction with $\mathrm{ClO}^{-}$(Sun et al., 2014). In particular, $\mathrm{H}_{2} \mathrm{O}_{2}$, a common cellular ROS that has a long in vivo half-life and can be generated at markedly high concentrations (up to low $\mathrm{mM}$ ) in certain pathophysiological conditions (Brewer et al., 2015), represents a major species competing for probe reactivity in a complex cellular milieu, confounding the unambiguous detection of $\mathrm{ONOO}^{-}$. Finally, existing probes-predominately in the form of small molecule dye derivatives-often require organic synthesis and lack the capability to be genetically encoded, a feature that would greatly lower the barrier to the widespread adoption of these probes by other laboratories. A genetically encodable sensor (Chen et al., 2017) would also facilitate cell-/tissue-specific expression for in vivo studies, as exemplified by the popular genetically encoded calcium sensors (e.g. GCaMP series (Lin and Schnitzer, 2016)). 
We previously developed pnGFP (Chen et al., 2013), the first and only genetically encoded fluorescent probe for the selective detection of peroxynitrite, by site-specific incorporation of $p$ boronophenylalanine $(p \mathrm{BoF})$ into the chromophore of a circularly permuted superfolder green fluorescent protein (cpsGFP). This is achieved via genetic code expansion (Chin, 2017; Liu and Schultz, 2010), an evolutionary technique that uses an orthogonal aminoacyl-tRNA synthetase (aaRS)-tRNA pair to direct the incorporation of a ncAA into proteins in response to a nonsense codon (often the amber UAG codon). Interestingly, we serendipitously discovered that the boronic acid-based chromophore of pnGFP shows unusual high selectivity for $\mathrm{ONOO}^{-}-$ essentially nonresponsive to even $1 \mathrm{mM} \mathrm{H}_{2} \mathrm{O}_{2}$ (> 2000 eq probe concentration). Mechanistic studies using site-directed mutagenesis, X-ray crystallography, ${ }^{11} \mathrm{~B}$ NMR, and computational simulation collectively revealed that the boron atom in pnGFP is converted to an $s p^{3}$-hybridized form by a nearby histidine residue via a polarized water bridge (Chen et al., 2016b). The unique pathway by which pnGFP gains high selectivity suggests that the protein scaffold of genetically encoded probes can be engineered to tune their chemoselectivity, which remains challenging to obtain from small molecule probes.

Here, using directed evolution, rational mutagenesis, and targeted reactivity screening guided by the crystal structure of pnGFP1.5-Y.Cro (Chen et al., 2016b), we engineered a high-performance peroxynitrite probe, pnGFP-Ultra, which shows ultra-high sensitivity and selectivity for $\mathrm{ONOO}^{-}$. Compared to pnGFP, pnGFP-Ultra has better chromophore maturation, brighter fluorescence, and a 6-fold enhancement on both sensitivity and selectivity, making it the most advanced genetically encoded biosensor for peroxynitrite. To facilitate the use of pnGFP-Ultra in mammalian cells, we systematically optimized the plasmid-based expression system for ncAA incorporation, leading to a 13.3-fold higher $p \mathrm{BoF}$-incorporated protein expression in HEK 293T cells. We demonstrate that pnGFP-Ultra can be used to robustly detect physiological peroxynitrite production in activated macrophages and primary mouse glia. Together, these developments make pnGFP-Ultra a powerful tool for imaging peroxynitrite, especially in experiments where genetic encodability and high selectivity are of primary interests.

\section{Results}

Design, Engineering, and Characterization of pnGFP1.5 
pnGFP was engineered by replacing the chromophore tyrosine residue of a circularly permutated superfolder green fluorescent protein (cpsGFP) with a ncAA, $p$ BoF (Chen et al., 2013). Upon reaction with $\mathrm{ONOO}^{-}$, the boronic acid-based chromophore (dark) is converted into its phenolate form (bright), leading to potent fluorescence turn-on response (Figure 1B). Despite the conceptual success, the broad utility of pnGFP is stymied by several of its drawbacks, including poor protein folding/chromophore maturation, low mammalian cell expression, and dim fluorescence before and after $\mathrm{ONOO}^{-}$conversion. Given the sensing mechanism of pnGFP, we aimed to first improve the folding, expression, and brightness of cpsGFP (Chen and Ai, 2014), the parental template of pnGFP that has a tyrosine derived chromophore (Figure S1). We carried out multiple rounds of directed protein evolution followed by colony-based screening in Escherichia coli $($ E. coli) cells (Figures S1A), leading to a brighter mutant, cpsGFP2 (cpsGFPV24A K27Q Y98H S136R) (Figure S1B). Next, we substituted the chromophore tyrosine residue of cpsGFP2 with $p \mathrm{BoF}(\mathrm{Y} 174 \mathrm{~B}$, where $\mathrm{B}=p \mathrm{BoF})$ through genetic code expansion. To screen for mutants with high reactivity and selectivity toward $\mathrm{ONOO}^{-}$, we performed saturation mutagenesis on threonine 5 (T5) and threonine 253 (T253), two terminal residues that have previously been shown to influence the reactivity of ncAA-containing fluorescent biosensors (Chen and Ai, 2014; Chen et al., 2012, 2013, 2016b). Library screening coupled with fluorescence-based in vitro assays (Figure S1) resulted in pnGFP1.5 (cpsGFP2-T5L Y174B T253S) (Figure 2), which exhibited a remarkable 132-fold fluorescence turn-on response towards $100 \mu \mathrm{M} \mathrm{ONOO}^{-}$(Figure 3A). pnGFP1.5 has a higher protein expression level in E. coli as compared to pnGFP, presumably because of enhanced protein folding and chromophore maturation.

\section{Structure-Guided Engineering of pnGFP-Ultra}

Compared to pnGFP, pnGFP1.5 showed a 6-fold enhancement in the dynamic range towards $\mathrm{ONOO}^{-}$(Figure 3A), but also an undesirable $\sim 3$-fold increase in the dynamic range towards $\mathrm{H}_{2} \mathrm{O}_{2}$ (Figure 3B). The overall selectivity (arbitrarily defined as fold of fluorescence enhancement by $\mathrm{ONOO}^{-}$/ fold of fluorescence enhancement by $\mathrm{H}_{2} \mathrm{O}_{2}$ ) of pnGFP1.5 remains higher than that of pnGFP (Figure 3C). We next sought to tune the selectivity of pnGFP1.5 by rational mutagenesis. In our previous attempt to decipher the unusual chemoselectivity of pnGFP, we solved the X-ray crystal structure of pnGFP1.5-Y.Cro (pnGFP1.5-B174Y, PDB 5F9G), which contains a tyrosine-derived chromophore (Chen et al., 2016b). The structure indicates that 
three residues - namely Thr64, Ser66, His9-are in close proximity to the phenolate oxygen of the chromophore (Figure 1C). We speculate that these residues may interact with the $p \mathrm{BoF}$ derived chromophore in pnGFP1.5 to fine tune the chromophore environment (electrostatics, hydrophobicity, and solvent accessibility). In addition, the first chromophore-forming residue (Thr173) can also affect electron distributions within the conjugate chromophore structure. Thus, these four residues are next subjected to site-directed mutagenesis (Figure 2 and S1). Based on our prior experiences with pnGFP, we created a panel of pnGFP1.5 mutants and assayed their selectivity in vitro (Figure S2). From this screening, we identified pnGFP1.6 (pnGFP1.5-S66A), which showed much lower reactivity towards $\mathrm{H}_{2} \mathrm{O}_{2}$, but maintained the same amplitude of response towards $\mathrm{ONOO}^{-}$. All other mutants exhibited lower selectivity than pnGFP1.5, either due to low fluorescence intensity after $\mathrm{ONOO}^{-}$conversion or high reactivity towards $\mathrm{H}_{2} \mathrm{O}_{2}$ (Figure S2). We next focused on improving pnGFP1.6 (Figure S1B).

pnGFP gains selectivity, in part, by leveraging the nucleophilic attack efficiency differences between $\mathrm{ONOO}^{-}$and $\mathrm{HOO}^{-}$(Chen et al., 2016b). Due to $p \mathrm{Ka}$ differences (11.6 for $\mathrm{H}_{2} \mathrm{O}_{2}$ and 6.8 for $\mathrm{ONOOH}$ ), the portion of $\mathrm{HOO}^{-}$at neutral $\mathrm{pH}$ is much lower than that of $\mathrm{ONOO}^{-}$, thereby disfavoring the reaction of pnGFP with $\mathrm{H}_{2} \mathrm{O}_{2}$. However, this difference alone is not sufficient to explain the remarkable selectivity of pnGFP, suggesting that the protein scaffold contributes to further reducing the effective local concentration of $\mathrm{HOO}^{-}$available for reaction with the $p$ BoF-derived chromophore. To this end, we substituted threonine 5-which gates an opening towards the chromophore-with two randomized residues by using degenerate NNK codons. Screening of the resultant library against both $\mathrm{H}_{2} \mathrm{O}_{2}$ and $\mathrm{ONOO}^{-}$led to pnGFP-Ultra (pnGFP1.6-T5KK, Figure 2 and S1B), which showed virtually no response to $100 \mu \mathrm{M} \mathrm{H}_{2} \mathrm{O}_{2}$ even after 1-hour incubation (Figure 3B). While this came at a price of a slightly reduced reactivity to $\mathrm{ONOO}^{-}$(Figure 3A), the selectivity of pnGFP-Ultra is 1.9- and 6-fold that of pnGFP1.6 and pnGFP, respectively (Figure 3C). Interestingly, in pnGFP-Ultra, threonine 5 is replaced with two consecutive lysine residues (Figure 2), which possibly abolish the reactivity of pnGFP-Ultra towards $\mathrm{H}_{2} \mathrm{O}_{2}$ by channeling a positively charged entry route that diminishes local availability of $\mathrm{H}_{2} \mathrm{O}_{2}$ near the $p \mathrm{BoF}$-derived chromophore. This unexpected screening result further highlights the unique scaffolding effect of protein-based sensors, which could be harnessed to achieve unusual chemoselectivity. 
We tested in vitro-purified pnGFP-Ultra against a panel of redox-active molecules, including ROS/RNS and thiols that are commonly present in a complex cellular milieu (Figure 3D). Except for $\mathrm{ONOO}^{-}$, none of the species surveyed triggered appreciable fluorescent changes, confirming that pnGFP-Ultra is highly selective towards $\mathrm{ONOO}^{-}$(Figure 3D). Immediately (less than 10 seconds) upon incubation with $100 \mu \mathrm{M} \mathrm{ONOO}^{-}$, pnGFP-Ultra displayed a robust 123fold fluorescence enhancement. By contrast, 1-hour incubation of pnGFP-Ultra with $100 \mu \mathrm{M}$ and $1 \mathrm{mM} \mathrm{H}_{2} \mathrm{O}_{2}$ triggered only 1.31- and 2.46-fold fluorescence increase, respectively (Figure 3D). Given the low basal fluorescence intensity of pnGFP-Ultra (essentially dark), these small fluorescence increases are negligible in most applications. The fluorescence intensity of pnGFPUltra increased linearly from high nanomolar to low micromolar $\mathrm{ONOO}^{-}$concentrations, with the limit of detection determined to be $277 \mathrm{nM}$ (Figure 3E). Compared to pnGFP, pnGFP-Ultra exhibits an overall 6-fold improvement in both the dynamic range and selectivity (Figure 3A and 3C). Taken together, these results demonstrate that pnGFP-Ultra is a high performance RNS biosensor that is highly sensitive and selective for peroxynitrite.

\section{Development of an Enhanced ncAA Incorporation System in Mammalian Cells}

Efficient co-translational ncAA incorporation in response to the amber TAG codon is crucial for the success of pnGFP-Ultra and other applications that require ncAA incorporation. Indeed, one of the fundamental limitations of pnGFP and other ncAA-based biosensors is their inadequate expression in mammalian cells. While ncAA incorporation in E. coli. has been thoroughly optimized to an extent that ncAA incorporation can reach a level comparable to that of canonical amino acids, mammalian incorporation of ncAA has proven to be far more challenging, presumably due to a more complicated ribosomal translation machinery.

Existing plasmid-based systems for ncAA incorporation (Chen et al., 2009; Hino et al., 2012; Liu et al., 2007; Schmied et al., 2014; Xiao et al., 2013) vary in the type and copy number of the engineered orthogonal tRNA-aminoacyl tRNA synthetase pair, and the choices of vectors and promoters, making it difficult to cross compare the efficiencies of these systems. Several strategies for enhancing amber codon suppression efficiency such as increasing the copy number of tRNAs (Liu et al., 2007) and ectopic expression of a dominant-negative eukaryotic release factor 1 mutant (eRF1-E55D) (Schmied et al., 2014) have proven to be generalizable. To systematically optimize ncAA incorporation into proteins in mammalian cells, we constructed a series of expression plasmids (plasmids $a-e$, Figure 4A) and tested ncAA incorporation in 
HEK293T cells using an EGFP $_{\mathrm{TAG}}$ fluorescent reporter gene that bears an amber TAG codon at position 39 of EGFP. Incorporation of a ncAA in response to the amber TAG codon (amber suppression) would generate the full length EGFP while failure to do so would result in a truncated EGFP (non-fluorescent). Previously, $p \mathrm{BoF}$ was incorporated into pnGFP by cotransfecting the CMV promoter-driven pnGFP reporter plasmid with the suppressor plasmid, which harbors one copy of CMV promoter-driven poly-specific aminoacyl tRNA synthetase (Poly-aaRS) and four copies of U6/H1 promoter-driven suppressor tRNAs (Figure 5A). Since orthogonal tRNA expression is a limiting factor in ncAA incorporation efficiency, we cloned four copies of tRNAs on the reporter plasmid $c$ as well (Figure 4A). Compared to our previous expression method using plasmids $a+b$, addition of extra copies of tRNA on the reporter plasmid doubled the incorporation efficiency of $p \mathrm{BoF}$ (Figure $4 \mathrm{~B}$ ) into the $\mathrm{EGFP}_{\mathrm{TAG}}$ reporter (Figure 4C, plasmids $c+b$ ). To test whether ectopic expression of eRF1-E55D further enhances ncAA incorporation efficiency, eRF1-E55D was bicistronically expressed with Poly-aaRS via a self-cleaving T2A peptide linker (Figure 4A, plasmid $d$ ). Co-expression of eRF1-E55Dcontaining suppressor plasmid $d$, in lieu of $b$, with the original reporter plasmid $a$ resulted in a 10.9-fold enhancement in $p \mathrm{BoF}$ incorporation (Figure 4C). Remarkably, we observed further improvements in incorporation efficiency using plasmids $c+d$, suggesting that the benefit from expression of extra copies of tRNA and the eRF1-E55D mutant are synergistic (Figure 4C). To simply the expression system, we integrated all genetic components necessary for ncAA incorporation-including the reporter gene, Poly-aaRS, and orthogonal tRNAs-into a single plasmid (Figure 4A, plasmid $e$ ). Expression of plasmid $e$ alone gave rise to comparable incorporation efficiency to that of using $a+d$. Co-transfection of plasmid $e$ with other plasmids led to various degrees of expression efficiency. Nevertheless, all strategies tested surpass our original method of using plasmids $a+b$ for ncAA incorporation, with plasmids $c+d$ and $c+e$ having the highest incorporation efficiency for $p \mathrm{BoF}$ (13.3-fold enhancement) (Figure 4C). We achieved similar enhancement in incorporation efficiency for $p$-azido-phenylalanine ( $p \mathrm{AzF}$ ) (Figure S3), suggesting that this plasmid-based system is quite general and can be used to incorporate other ncAAs.

\section{Imaging of Peroxynitrite in Live Cells using pnGFP-Ultra}

Next, we constructed a mammalian expression plasmid by replacing the EGFP $_{\text {TAG }}$ gene with pnGFP-Ultra gene in plasmid $c$ (Figure 5A). We co-expressed the resultant plasmid with plasmid 
$d$, as this combination gave rise to the highest $p \mathrm{BoF}$ incorporation efficiency in the above $\mathrm{EGFP}_{\mathrm{TAG}}$ reporter assay (Figure 4C). To compare with the first-generation biosensor, we expressed pnGFP in parallel by utilizing our previous method (Figure 5A). After treatment with SIN-1, a cell permeable $\mathrm{ONOO}^{-}$donor, the fluorescence intensities from pnGFP-Ultraexpressing cells were much higher than those from pnGFP-expressing cells (Figure 5B). It is also evident (from the number of fluorescent cells) that the expression efficiency of pnGFP-Ultra is much higher than that of pnGFP. Both pnGFP and pnGFP-Ultra are selective towards $\mathrm{ONOO}^{-}$, with no obvious response toward $\mathrm{H}_{2} \mathrm{O}_{2}(1 \mathrm{mM})$ or $\mathrm{HOCl}(100 \mu \mathrm{M})$ (Figure $\left.\mathrm{S} 4\right)$. We attribute the superior performance of pnGFP-Ultra to both its large dynamic range (123-fold fluorescence enhancement) and the substantially improved $p \mathrm{BoF}$ incorporation. Together, these developments make pnGFP-Ultra a high-performance biosensor for imaging $\mathrm{ONOO}^{-}$in mammalian cells.

\section{Visualization of Peroxynitrite Production in Activated Macrophages}

To further demonstrate the utility of pnGFP-Ultra in imaging physiological peroxynitrite, we expressed pnGFP-Ultra in mouse RAW264.7 macrophage immune cells, which are known to produce $\mathrm{ONOO}^{-}$for microbicidal activities under immune stimulation (Allen et al., 2012; Peng et al., 2014; Xia and Zweier, 1997). Upon co-stimulation with bacterial endotoxin lipopolysaccharide (LPS) and pro-inflammatory cytokine interferon-gamma (IFN- $\gamma$ ), we observed strong fluorescence signal in pnGFP-Ultra expressing cells (Figure 6A). The mean fluorescence of cells in the treated group was $~ 3.7$-fold higher than that of untreated cells (Figure 6B), suggesting that pnGFP-Ultra can reliably detecting physiological peroxynitrite production.

\section{Detection of Peroxynitrite Production in Amyloid ß-Activated Primary Mouse Glia}

Despite the continuous improvement in life expectancy, Alzheimer's disease (AD) and other neurodegenerative disorders have become a global health and social challenge (Association, 2019). Peroxynitrite is known to play important roles in numerous neurodegenerative diseases. For example, peroxynitrite-mediated damage has been broadly observed in AD (Smith et al., 1997) and the amyloid $\beta$ peptide, whose accumulation has been considered to be the hallmark of $\mathrm{AD}$, is known to induce peroxynitrite production in glial cells (González-Reyes et al., 2017; Xie et al., 2002) and subsequently mediate neurotoxicity (Xie et al., 2002). To test whether pnGFPUltra can detect peroxynitrite production in neurological systems, we expressed pnGFP-Ultra in 
cultured primary mouse glia cells. Indeed, we observed dramatically enhanced fluorescence signal from glia activated with amyloid $\beta$ (Figure 7A), producing a $\sim 3$.8-fold mean fluorescence enhancement compared with untreated cells (Figure 7B). Thus, pnGFP-Ultra represents a novel tool enabling the imaging of peroxynitrite production in neurological systems. In addition, it opens up an exciting possibility to develop pnGFP-Ultra-based fluorescent assays for highthroughput screening of inhibitors that targets major $\mathrm{ONOO}^{-}$production pathways. Such capability will aid the development of drugs that protect neurons against $\mathrm{ONOO}^{-}$-mediated cell toxicity/death (Szabó et al., 2007).

\section{Discussion}

In summary, we have developed pnGFP-Ultra - a highly sensitive and selective fluorescent peroxynitrite biosensor-by directed evolution, structure-guided rational design, and reactivitybased library screening. Compared to the first-generation peroxynitrite probe pnGFP, pnGFPUltra has much better chromophore maturation and a 6-fold larger $\mathrm{ONOO}^{-}$-induced fluorescence response, while retaining the high chemoselectivity against $\mathrm{H}_{2} \mathrm{O}_{2}$ and other ROS/RNS. An apparent hurdle of applying ncAA-based biosensors to cell imaging studies has been the inefficiency with which ncAA can be incorporated into proteins in mammalian cells. Here, we systematically optimized and increased $p$ BoF incorporation efficiency in HEK 293T cells by 13.3-fold. These developments make pnGFP-Ultra a high-performance biosensor capable of imaging physiological peroxynitrite, as we have demonstrated in activated macrophages and primary glial cells. pnGFP-Ultra thus represents an important advancement enabling the practical implementation of a genetically encoded biosensor for live-cell detection of peroxynitrite. We expect pnGFP-Ultra to find broad utility in probing RNS biology in diverse pathophysiological settings.

pnGFP-Ultra not only fills an important technological gap in the field of RNS, but also conveys innovations that will inspire the development of future biosensors for other bioanalytical targets. First, the chromophore (and other residues) of circularly permutated fluorescent proteins can be modified into a hub of chemical sensing, binding, reactivity, and catalysis, thereby greatly expanding the use of fluorescent proteins as reporters. Second, direct evolution and rational mutagenesis performed on noncanonical amino acid-modified proteins can lead to new and sometimes, surprising functions, as we have demonstrated here via the engineering of exquisite chemo-selectivity of pnGFP-Ultra. 
Compared to existing small-molecule based peroxynitrite probes, pnGFP-Ultra has the advantage of being genetically encodable. This feature permits long-term in vivo imaging and organelle-/cell-/tissue-specific targeting, making it possible to apply pnGFP-Ultra to a variety of biological/disease models. More importantly, because of the convenient fluorescence readout and robust off-on response of pnGFP-Ultra, cell lines stably expressing this reporter can be used in high-throughput drug screening assays. pnGFP-Ultra may also be used in genome-wide CRISPR screens (Shalem et al., 2015) to uncover new genes involved in $\mathrm{NO} \bullet, \mathrm{O}_{2}^{-\bullet}$, or $\mathrm{ONOO}^{-}$production pathways. In addition to live cell imaging, we envision these high-throughput drug/genetic screens to be an important avenue of application where pnGFP-Ultra shall prove to be an enabling tool. Since pnGFP-Ultra can be widely distributed in the form of plasmids, it represents a valuable catalyst to future breakthroughs in the field of RNS.

\section{STAR田Methods}

Key Resources Table

\begin{tabular}{|c|c|c|}
\hline REAGENT or RESOURCE & SOURCE & IDENTIFIER \\
\hline \multicolumn{3}{|c|}{ Chemicals, Peptides, and Recombinant Proteins } \\
\hline p-borono-DL-phenylalanine & Synthonix & $\begin{array}{l}\text { Cat\#: B5001; } \\
\text { CAS:90580-64-6 }\end{array}$ \\
\hline$p$-azido-phenylalanine & Bachem & $\begin{array}{l}\text { Cat\#: F-4305; } \\
\text { CAS:1241681-80-0 }\end{array}$ \\
\hline Sodium peroxynitrite & Cayman Chemical & $\begin{array}{l}\text { Cat\#: } 81565 ; \\
\text { CAS: } 14042-01-4\end{array}$ \\
\hline $\begin{array}{l}\text { 3-morpholinosydnonimine (SIN- } \\
\text { 1) }\end{array}$ & Sigma Aldrich & $\begin{array}{l}\text { Cat\#: M5793; } \\
\text { CAS:16142-27-1 }\end{array}$ \\
\hline $\begin{array}{l}\text { Human Interferon- } \gamma \text { Protein, } \\
\text { recombinant }\end{array}$ & Sigma Aldrich & Cat\#: IF002 \\
\hline
\end{tabular}




\begin{tabular}{|l||l||l|}
\hline \hline REAGENT or RESOURCE & SOURCE & IDENTIFIER \\
\hline $\begin{array}{l}\text { Lipopolysaccharides (LPS) } \\
\text { from Salmonella typhosa }\end{array}$ & Sigma Aldrich & Cat\#: L7895 \\
\hline$\beta$-Amyloid (1-42), human & GenScript & Cat\#: RP10017 \\
\hline
\end{tabular}

\section{Critical Commercial Assays}

\begin{tabular}{|l||l||l|}
\hline B-PER & Thermo Fisher Scientific & Cat\#: 78243 \\
\hline TRI Reagent & Thermo Fisher Scientific & Cat\#: AM9738 \\
\hline \hline $\begin{array}{l}\text { SuperScript@ IV Reverse } \\
\text { Transcriptase }\end{array}$ & Thermo Fisher Scientific & Cat\#: 18090010 \\
\hline RIPA Lysis and Extraction Buffer & Thermo Fisher Scientific & Cat\# 89900 \\
\hline
\end{tabular}

\section{Cell Culture Reagents}

\begin{tabular}{|l|l||l||}
\hline $\begin{array}{l}\text { Dulbecco's modified Eagle's } \\
\text { medium (DMEM) }\end{array}$ & Corning & Cat\# 10-017-CV \\
\hline \hline Trypsin EDTA & Corning & Cat\# 25-053-CI \\
\hline $\begin{array}{l}\text { Fetal Bovine Serum } \\
\text { Penicillin-Streptomycin Solution, }\end{array}$ & CenClone & Cat\# 25-550 \\
\hline \hline $\begin{array}{l}\beta \text {-mercaptoethanol } \\
\text { DPBS }\end{array}$ & MP Biomedicals & Cat\# 30-002-CI \\
\hline Cat\# 773648 $60-24-2$ \\
\hline
\end{tabular}

\section{Cell Transfection Reagents}




\begin{tabular}{|l|l|l|}
\hline REAGENT or RESOURCE & SOURCE & IDENTIFIER \\
\hline Viromer & ORIGENE & Cat\# TT100312 \\
\hline Lipofectamine 2000 & Thermo Fisher Scientific & Cat\# 11668027 \\
\hline
\end{tabular}

\section{Data}

\begin{tabular}{|l|l|l} 
MS source data & This paper & Available upon request \\
\hline
\end{tabular}

\section{Experimental Models: Cell Lines}

\begin{tabular}{|c|c|c|}
\hline Human HEK 293T cells & ATCC & CRL-3216 ${ }^{\mathrm{TM}}$ \\
\hline $\begin{array}{l}\text { Escherichia coli } \mathrm{DH} 10 \mathrm{~B} \\
\text { competent cells }\end{array}$ & Thermo Fisher Scientific & Cat\# 18297010 \\
\hline RAW264.7 Macrophage cells & ATCC & TIB-71 ${ }^{\mathrm{TM}}$ \\
\hline
\end{tabular}

\section{Recombinant DNA}

\begin{tabular}{|l|l|l|}
\hline Synthetic oligo nucleotides & IDT & Table S1 \\
\hline pUltra & (Lou et al., 2012) & Addgene \# 24129 \\
\hline pMAH-POLY & (Chen et al., 2013) & Addgene \# 64915 \\
\hline pcDNA3-pnGFP & (Chen et al., 2013) & Addgene \# 64913 \\
\hline pEvol-BoF & (Young et al., 2010) & N/A \\
\hline pBAD-cpsGFP2 & This paper & N/A \\
\hline pBAD-pnGFP-Ultra & This paper & Addgene \#157923 \\
\hline
\end{tabular}




\begin{tabular}{|l|l|l||}
\hline REAGENT or RESOURCE & SOURCE & IDENTIFIER \\
\hline pcDNA3-EGFP $_{\text {TAG }}$ & This paper, Figure 4A, a & N/A \\
\hline pMAH-EGFP \\
TAG & This paper, Figure 4A, c & N/A \\
\hline pMAH-POLY-eRF1 $1_{\text {E55D }}$ & This paper, Figure 4A, d & Addgene \#157925 \\
\hline pMAH-EGFP TAG $_{\text {POLY }}$ & This paper, Figure 4A, e & N/A \\
\hline pMAH-pnGFP-Ultra & This paper, Figure 5A & Addgene \#157924 \\
\hline Softwares and Algorithms & (Schindelin et al., 2012) & $\underline{\text { https://fiji.sc }}$ \\
\hline Fiji & Schrödinger & $\underline{\text { https://pymol.org/2/ }}$ \\
\hline PyMOL & GraphPad & $\underline{\underline{\text { https://www.graphpad.co }}}$ \\
\hline GraphPad Prism 8 & $\underline{\text { software/prism/ }}$ \\
\hline
\end{tabular}

\section{Resource Availability}

\section{Lead Contact}

Further information and requests for resources and reagents should be directed to and will be fulfilled by the Lead Contact, Huiwang Ai (huiwang.ai@ virginia.edu).

\section{Materials Availability}

All reagents created in this study (see Key Resources Table) are available on request. Key plasmids generated have been deposited to Addgene.

\section{Data Availability}


Raw source data are available upon reasonable request.

\section{Experimental Model and Subject Details}

HEK 293T cells (human, female, embryonic kidney) were maintained in Dulbecco's modified Eagle's medium (DMEM) supplemented with $10 \%$ (v/v) FBS at $37^{\circ} \mathrm{C}$ and $5 \% \mathrm{CO}_{2}$ atmosphere. Other cell types were cultured as described in Supplementary Experimental Procedures.

\section{Methods Details}

\section{Materials and General Methods}

Synthetic DNA oligonucleotides were purchased from Integrated DNA Technologies (San Diego, CA). Restriction endonucleases were purchased from Thermo Fisher Scientific (Waltham, MA). PCR and restriction digestion products were purified by gel electrophoresis and extracted using the Syd Labs Gel Extraction kit (Malden, MA). Plasmid DNA was purified using the Syd Labs Miniprep kit (Malden, MA). DNA sequencing was analyzed by Retrogen (San Diego, CA). The amino acid $p$-borono-DL-phenylalanine ( $p \mathrm{BoF}$ ) was purchased from Synthonix (Wake Forest, NC). Fluorescence measurements were performed on a monochromator-based Synergy Mx Microplate Reader (BioTek, Winooski, VT). Mammalian cell imaging was performed on a Leica SP5 confocal fluorescence microscope or a Leica DMi8 microscope equipped with a Photometrics Prime 95B sCMOS camera. Other materials and general procedures were acquired or performed as previously described (Chen et al., 2012, 2013, 2016b).

\section{Engineering and Screening of pnGFP1.5}

To improve the brightness and folding of pnGFP, several rounds of error prone PCR (EP-PCR) based directed evolution were performed to improve its template-cpsGFP (Chen and Ai, 2014), which contains a tyrosine-derived chromophore. Briefly, oligos pBAD-FP and pBAD-RP were used to amplify the cpsGFP gene from the pBAD-cpsGFP plasmid in an EP-PCR reaction condition. The mutated PCR products were digested with XhoI and HindIII and ligated into a predigested compatible pBAD vector. The ligated product was used to transform Escherichia coli DH10B competent cells by electroporation. Cells were grown on Luria-Bertani (LB) broth agar plates supplemented with $100 \mu \mathrm{g} / \mathrm{mL}$ ampicillin and $0.02 \%$ L-arabinose. LB agar plates were incubated at $37^{\circ} \mathrm{C}$ overnight. Bacterial colonies were illuminated under a laboratory-built 
colony fluorescence imaging system and examined using a pair of forensic yellow goggles with a cutoff wavelength of $\sim 490 \mathrm{~nm}$. Plasmid DNA from the brightest colonies of each round were extracted and combined to serve as the template for the next round of EP-PCR. The fluorescence of the B-PER (Pierce, Rockford, IL) extracted cell lysate from the brightest colonies of the final round was quantitatively compared on the Synergy Mx Microplate Reader. The best mutant was sequenced and renamed as cpsGFP2.

To screen for pnGFP1.5, the codon for the chromophore tyrosine (Tyr174) of cpsGFP2 were mutated to TAG to allow for $p \mathrm{BoF}$ incorporation using the pEvol-BoF-based amber suppression system. Next, threonine 5 (Thr5) and threonine 253 (Thr253) of cpsGFP2Tyr174TAG were randomized using NNK degenerate codons to construct a gene library. The library was used to screen for mutants with high reactivity to $\mathrm{ONOO}^{-}$. The chromophore TAG mutagenesis, library construction and screening procedures were the same as those in pnGFP engineering, which was detailed previously (Chen et al., 2013). The best mutant from the library was sequenced and designated as pnGFP1.5.

\section{Development and Characterization of pnGFP-Ultra}

To create the various pnGFP1.5 mutants, site-directed mutagenesis of pnGFP1.5 was performed using an overlap extension PCR based strategy. The procedures were described previously (Chen et al., 2016b), and the mutagenesis primers can be found in Supplementary Information Table S1. Proteins from each mutant were purified, buffer-exchanged and concentrated as described. To test the reactivity and selectivity of the pnGFP1.5 mutants, $0.2 \mu \mathrm{M}$ purified protein was incubated with Tris buffer (150 mM Tris- $\mathrm{HCl}, 150 \mathrm{mM} \mathrm{NaCl}, \mathrm{pH} 7.4), 100 \mu \mathrm{M} \mathrm{ONOO}^{-}$or 100 $\mu \mathrm{M} \mathrm{H}_{2} \mathrm{O}_{2}$ for 1 hour at room temperature in Tris buffer. Fluorescence intensities at $510 \mathrm{~nm}$ with a $490 \mathrm{~nm}$ excitation were quantified and represented as means \pm SD from three independent measurements. Fluorescence enhancements were calculated by dividing the fluorescence of the $\mathrm{ONOO}^{-}$- or $\mathrm{H}_{2} \mathrm{O}_{2}$-treated group by that of the control group at the 1-hour time point ( $\mathrm{DR}_{\mathrm{ONOO}-}$ or $\left.\mathrm{DR}_{\mathrm{H} 2 \mathrm{O} 2}\right)$. The selectivity of each mutant was defined as the ratio of the fluorescence enhancement from $\mathrm{ONOO}^{-}$-treated group over that from $\mathrm{H}_{2} \mathrm{O}_{2}$-treated group $\left(\mathrm{DR}_{\mathrm{ONOO}-} /\right.$ $\mathrm{DR}_{\mathrm{H} 2 \mathrm{O} 2}$ ). The mutant with the highest selectivity, namely pnGFP1.5-S66A (pnGFP1.6), was chosen for further engineering.

To screen for mutants with further enhanced selectivity, the threonine 5 (Thr5) codon of pnGFP1.6 was mutated into two consecutive degenerative codons (NNKNNK) using primers 
pnGFP1.6-F-NNK2 and pBAD-RP. The gene library was screened and constructed as described before. The mutant with the highest selectivity from this library was sequenced and designated as pnGFP-Ultra. Characterization protocols for pnGFP-Ultra including the purification, reactivity measurements, selectivity tests over other reactive chemical species, and determination of the limits of detection (LOD) were the same as those for pnGFP (Chen et al., 2013).

\section{Construction of Mammalian Expression Plasmids}

Plasmid $a$ and $b$ were previously described (Chen et al., 2013). To construct plasmid $c$, the EGFP $_{\text {TAG }}$ gene was amplified from plasmid $a$ with oligos EGFP-F and EGFP-R. The PCR product was digested with HindIII and ApaI, and ligated into plasmid $b$, which was predigested with the same enzymes. To construct plasmid $d$, the eRF1 gene was first cloned from the total cDNA of HEK 293T cells with oligos eRF1-F and eRF1-R. To prepare the cDNA, total mRNA from HEK293T cell was isolated with the TRI Reagent (Sigma, St. Louis, MO) and reversetranscribed with the SuperScript ${ }^{\circledR}$ IV Reverse Transcriptase (Thermo Fisher Scientific, Carlsbad, CA). To introduce the E55D point mutation within eRF1, oligos eRF1-NheI-F and E55D-R, E55D-F and eRF1-EcoRI-R were used to amplify the two overlapping fragments flanking E55 position of eRF1, respectively. Next, the two fragments were joined by overlap extension PCR with oligos eRF1-NheI-F and eRF1-EcoRI-R, and sub-cloned into the pUltra vector (Addgene Plasmid \#24129) between restriction sites NheI and EcoRI to generate pUltra-eRF1-E55D, which contains the T2A-eRF1-E55D-WPRE cassette. This cassette was amplified with oligos eRF1-GB-F and eRF1-GB-R and ligated into ApaI-linearized plasmid $b$ using Gibson assembly cloning (Gibson et al., 2009). To construct plasmid $e$, the CMV- EGFP amplified from plasmid $a$ with oligos CMV-39TAG-XhoI-F and CMV-39TAG-XhoI-R. The PCR product was digested with XhoI and ligated into XhoI digested, DpnI dephosphorylated plasmid $b$. To construct the mammalian expression plasmid for pnGFP-Ultra, the pnGFP-Ultra gene was amplified from pBAD-pnGFP-Ultra with oligos pnGFP-Ultra-HindIII-F and pnGFPUltra-ApaI-R, and sub-cloned into plasmid $c$ between restriction sites Hind III and ApaI.

\section{Mammalian Cell Culture, Transfection and Imaging}

Human Embryonic Kidney (HEK) 293T cells were cultured in Dulbecco's Modified Eagle's Medium (DMEM) supplemented with $10 \%$ fetal bovine serum (FBS). Cells were incubated at $37^{\circ} \mathrm{C}$ with $5 \% \mathrm{CO}_{2}$ in humidified air. To test the transfection and non-canonical amino 
acid (ncAA) incorporation efficiency of the various plasmids, cells were split and seeded into 24well plates 16 hours before transfection. Cells were transfected with 600 ng DNA and $2 \mu \mathrm{g}$ PEI (polyethyleneimine, linear, M.W. $25 \mathrm{kDs}$ ) per well for three hours, and then cultured in complete medium supplemented with $2 \mathrm{mM}$ DL- $p$ BoF for 48 hours. Cells were lysed with $150 \mu \mathrm{L}$ RIPA buffer and centrifuged at $13,000 \mathrm{rpm}, 4^{\circ} \mathrm{C}$ for 5 mins. The fluorescence of the EGFP-containing supernatant $(100 \mu \mathrm{L})$ was measured on the microplate reader with excitation and emission wavelength set at $490 \mathrm{~nm}$ and $515 \mathrm{~nm}$, respectively. Transfections were triplicated and data were represented as mean $\pm \mathrm{SD}$.

To express pnGFP or pnGFP-Ultra, HEK $293 \mathrm{~T}$ cells in each 35-mm plastic culture dish were co-transfected with $1.5 \mu \mathrm{g}$ pnGFP-containing plasmid $a$ and $1.5 \mu \mathrm{g}$ plasmid $b$ or $1.5 \mu \mathrm{g}$ pnGFP-Ultra-containing plasmid $c$ and $1.5 \mu \mathrm{g}$ plasmid $d$, in the presence of $10 \mu \mathrm{g}$ PEI. Other procedures were the same as those for pnGFP (Chen et al., 2013). Cells were imaged under a Leica DMi8 microscope equipped with a Photometrics Prime 95B sCMOS camera. A GFP filter cube with a 470/40 $\mathrm{nm}$ bandpass excitation filter and a 525/50 $\mathrm{nm}$ bandpass emission filter was used for cell imaging.

\section{RAW 264.7 Macrophage Cell Culture, Transfection and Activation}

RAW 264.7 cells were obtained from ATCC and grown at $37^{\circ} \mathrm{C}$ under $5 \% \mathrm{CO}_{2}$ in $\mathrm{DMEM}$ supplemented with $10 \% \mathrm{FBS}, 100 \mu \mathrm{g} / \mathrm{mL}$ penicillin, $100 \mu \mathrm{g} / \mathrm{mL}$ streptomycin, and $58 \mu \mathrm{M} \beta$ mercaptoethanol. Cells were passaged $18 \mathrm{~h}$ prior to transfection, and next, transfected with 1.5 $\mu \mathrm{g}$ pnGFP-Ultra-containing plasmid $c$ and $1.5 \mu \mathrm{g}$ plasmid $d$ in the presence of Viromer according to the manufacturer's instruction. After transfection, cells were cultured in the aforementioned complete media supplemented with $2 \mathrm{mM} \mathrm{DL}-p$ BoF. $24 \mathrm{~h}$ after transfection, IFN- $\gamma(50 \mathrm{U} / \mathrm{ml})$ and LPS (100 ng/ml) were added into the cell culture media, and the incubation lasted for another $18 \mathrm{~h}$. Cells were next cultured in fresh complete media supplemented with IFN- $\gamma$ (50 $\mathrm{U} / \mathrm{ml})$ and LPS (100 ng/ml) in the absence of DL- $p$ BoF for additional $18 \mathrm{~h}$. Cells in the control group were untreated with IFN- $\gamma$ and LPS but otherwise handled in parallel. Cells were imaged under the same condition as used for imaging HEK 293T.

\section{Isolation and Culture of Primary Glial Cells}

Primary glial cells were isolated from the brain of newborn (0-2 days postnatal) wild-type BALB/C mice and cultured as previously described (Redlich et al., 2013; Seele et al., 2016). 
Briefly, meninges of mouse brains were removed and whole brains were cut into small pieces and treated with $0.25 \%$ trypsin $(5 \mathrm{~mL} /$ brain $)$ for $15 \mathrm{~min}$ at $37^{\circ} \mathrm{C}$. After centrifugation $(1000 \times \mathrm{g}$, $1 \mathrm{~min})$, trypsin was removed and DMEM (4.5 g/L glucose, $3 \mathrm{~mL} / \mathrm{brain})$ was added into the cell precipitation, which was further minced by pipetting up and down with a $1 \mathrm{~mL}$ pipette. After another centrifugation $(750 \times \mathrm{g}, 8 \mathrm{~min})$, the supernatant, which contained the needed cells, was filtered with a 40- $\mu \mathrm{m}$ sterile cell strainer (Nylon mesh, Fisherbrand, Cat\#: 22363547). Cells were next seeded onto $35 \mathrm{~mm}$ cell culture dishes with poly-D-lysine coated glass bottoms and cultured in DMEM (4.5 mg/L glucose) supplemented with 10\% FBS, $5 \mathrm{mM}$ glutamine and $100 \mathrm{U} / \mathrm{ml}$ penicillin and streptomycin at $37^{\circ} \mathrm{C}, 5 \% \mathrm{CO}_{2}$ for 8-10 days before transfection. This wellestablished procedure is known to derive mixed glial cells composed of astrocytes and microglia (Redlich et al., 2013; Seele et al., 2016).

\section{Transfection and Activation of Primary Glial Cells}

Primary glial cells were transfected with Lipofectamine 2000 by following the manufacturer's instruction. After transfection, cells were cultured in the complete culture media supplemented with $2 \mathrm{mM}$ DL-pBoF. $\beta \square$ amyloid (1-42, human) was added at a final concentration of $5 \mu \mathrm{M}$ at $24 \mathrm{~h}$ post transfection. The incubation lasted for another $24 \mathrm{~h}$ and the culture media were replaced with fresh complete media supplemented with $5 \mu \mathrm{M} \beta \square$ amyloid but without DL-pBoF. The treatment lasted for another $18 \mathrm{~h}$ before microscopic images were acquired under the same condition as used for imaging HEK 293T. Cells in the control group were untreated with $\beta \square$ amyloid but otherwise handled in parallel.

\section{Acknowledgements}

Research reported in this publication was supported in part by the National Science Foundation under Award CHE-1750660 and the National Institute of General Medical Science of National Institutes of Health under Awards R01GM118675 and R01GM129291 (including the Supplement Award 3R01GM129291-02S2 from the National Institute on Aging). The content is solely the responsibility of the authors and does not necessarily represent the official views of the funding agencies.

\section{Author Contributions}


H.W., Z.C., and S.Z. conceived and planned experiments; Z.C. and S.Z carried out experiments; X.L isolated and cultured mouse glia cells. Z.C., S.Z., and H.W performed data analysis and interpretation; Z.C. prepared figures and wrote the initial draft of the manuscript; Z.C., S.Z., and H.W. edited and approved the manuscript.

\section{Declaration of Interests}

The authors declare no conflict of interests.

\section{Supplemental Information}

Supplemental Information includes a supplementary table of oligonucleotides sequences used in this study and four supplementary figures and can be found with this article online at http://doi.org/xxx. 


\section{References}

Allen, R.G., Lafuse, W.P., Powell, N.D., Marketon, J.I.W., Stiner-Jones, L.M., Sheridan, J.F., and Bailey, M.T. (2012). Stressor-induced increase in microbicidal activity of splenic macrophages is dependent upon peroxynitrite production. Infect. Immun. 80, 3429-3437.

Association, A. (2019). 2019 Alzheimer's disease facts and figures. Alzheimer's Dement. 15, $321-387$.

Beckman, J.S. (2009). Understanding peroxynitrite biochemistry and its potential for treating human diseases. Arch. Biochem. Biophys. 484, 114-116.

Beckman, J.S., and Koppenol, W.H. (1996). Nitric oxide, superoxide, and peroxynitrite: The good, the bad, and the ugly. Am. J. Physiol. - Cell Physiol. 271.

Brand, M.D. (2016). Mitochondrial generation of superoxide and hydrogen peroxide as the source of mitochondrial redox signaling. Free Radic. Biol. Med. 100, 14-31.

Brewer, T.F., Garcia, F.J., Onak, C.S., Carroll, K.S., and Chang, C.J. (2015). Chemical Approaches to Discovery and Study of Sources and Targets of Hydrogen Peroxide Redox Signaling Through NADPH Oxidase Proteins. Annu. Rev. Biochem. 84, 765-790.

Chen, Z.J., and Ai, H.W. (2014). A Highly Responsive and Selective Fluorescent Probe for Imaging Physiological Hydrogen Sulfide. Biochemistry 53, 5966-5974.

Chen, P.R., Groff, D., Guo, J., Ou, W., Cellitti, S., Geierstanger, B.H., and Schultz, P.G. (2009). A facile system for encoding unnatural amino acids in mammalian cells. Angew. Chemie - Int. Ed. 48, 4052-4055.

Chen, S., Chen, Z.-J., Ren, W., and Ai, H.-W. (2012). Reaction-based genetically encoded fluorescent hydrogen sulfide sensors. J. Am. Chem. Soc. 134.

Chen, Z., Truong, T.M., and Ai, H.W. (2016a). Chapter 10: Development of fluorescent probes for the detection of peroxynitrite. In RSC Detection Science, (Royal Society of Chemistry), pp. 186-207. 
Chen, Z., Truong, T., and Ai, H. (2017). Illuminating Brain Activities with Fluorescent ProteinBased Biosensors. Chemosensors 5, 32.

Chen, Z.J., Ren, W., Wright, Q.E., and Ai, H.W. (2013). Genetically encoded fluorescent probe for the selective detection of peroxynitrite. J. Am. Chem. Soc. 135, 14940-14943.

Chen, Z.J., Tian, Z., Kallio, K., Oleson, A.L., Ji, A., Borchardt, D., Jiang, D.E., Remington, S.J., and Ai, H.W. (2016b). The N-B Interaction through a Water Bridge: Understanding the Chemoselectivity of a Fluorescent Protein Based Probe for Peroxynitrite. J. Am. Chem. Soc. 138, 4900-4907.

Chin, J.W. (2017). Expanding and reprogramming the genetic code. Nature 550, 53-60.

Ferrer-Sueta, G., and Radi, R. (2009). Chemical biology of peroxynitrite: Kinetics, diffusion, and radicals. ACS Chem. Biol. 4, 161-177.

Förstermann, U., and Münzel, T. (2006). Endothelial nitric oxide synthase in vascular disease: From marvel to menace. Circulation 113, 1708-1714.

Fridovich, I. (1997). Superoxide anion radical (O2/-•), superoxide dismutases, and related matters. J. Biol. Chem. 272, 18515-18517.

Gibson, D.G., Young, L., Chuang, R.Y., Venter, J.C., Hutchison, C.A., and Smith, H.O. (2009). Enzymatic assembly of DNA molecules up to several hundred kilobases. Nat. Methods 6, 343345.

González-Reyes, R.E., Nava-Mesa, M.O., Vargas-Sánchez, K., Ariza-Salamanca, D., and MoraMuñoz, L. (2017). Involvement of astrocytes in Alzheimer's disease from a neuroinflammatory and oxidative stress perspective. Front. Mol. Neurosci. 10, 427.

Hardy, M., Zielonka, J., Karoui, H., Sikora, A., Michalski, R., Podsiadły, R., Lopez, M., Vasquez-Vivar, J., Kalyanaraman, B., and Ouari, O. (2018). Detection and Characterization of Reactive Oxygen and Nitrogen Species in Biological Systems by Monitoring Species-Specific Products. Antioxidants Redox Signal. 28, 1416-1432. 
Hino, N., Sakamoto, K., and Yokoyama, S. (2012). Site-specific incorporation of unnatural amino acids into proteins in mammalian cells. Methods Mol. Biol. 794, 215-228.

Li, X., Tao, R.R., Hong, L.J., Cheng, J., Jiang, Q., Lu, Y.M., Liao, M.H., Ye, W.F., Lu, N.N., Han, F., et al. (2015). Visualizing Peroxynitrite Fluxes in Endothelial Cells Reveals the Dynamic Progression of Brain Vascular Injury. J. Am. Chem. Soc. 137, 12296-12303.

Liaudet, L., Vassalli, G., and Pacher, P. (2009). Role of peroxynitrite in the redox regulation of cell signal transduction pathways. Front. Biosci. 14, 4809-4814.

Lin, M.Z., and Schnitzer, M.J. (2016). Genetically encoded indicators of neuronal activity. Nat. Neurosci. 19, 1142-1153.

Lin, K.K., Wu, S.C., Hsu, K.M., Hung, C.H., Liaw, W.F., and Wang, Y.M. (2013). A N-(2aminophenyl)-5-(dimethylamino)-1-naphthalenesulfonic amide (Ds-DAB) based fluorescent chemosensor for peroxynitrite. Org. Lett. 15, 4242-4245.

Liu, C.C., and Schultz, P.G. (2010). Adding New Chemistries to the Genetic Code. Annu. Rev. Biochem. 79, 413-444.

Liu, W., Brock, A., Chen, S., Chen, S., and Schultz, P.G. (2007). Genetic incorporation of unnatural amino acids into proteins in mammalian cells. Nat. Methods 4, 239-244.

Lou, E., Fujisawa, S., Morozov, A., Barlas, A., Romin, Y., Dogan, Y., Gholami, S., Moreira, A.L., Manova-Todorova, K., and Moore, M.A.S. (2012). Tunneling nanotubes provide a unique conduit for intercellular transfer of cellular contents in human malignant pleural mesothelioma. PLoS One 7.

Mungrue, I.N., Gros, R., You, X., Pirani, A., Azad, A., Csont, T., Schulz, R., Butany, J., Stewart, D.J., and Husain, M. (2002). Cardiomyocyte overexpression of iNOS in mice results in peroxynitrite generation, heart block, and sudden death. J. Clin. Invest. 109, 735-743.

Nadler, A., and Schultz, C. (2013). The power of fluorogenic probes. Angew. Chemie - Int. Ed. $52,2408-2410$. 
Pacher, P., Beckman, J.S., and Liaudet, L. (2007). Nitric oxide and peroxynitrite in health and disease. Physiol. Rev. 87, 315-424.

Peng, T., and Yang, D. (2010). HKGreen-3: A rhodol-based fluorescent probe for peroxynitrite. Org. Lett. 12, 4932-4935.

Peng, T., Wong, N.K., Chen, X., Chan, Y.K., Ho, D.H.H., Sun, Z., Hu, J.J., Shen, J., El-Nezami, H., and Yang, D. (2014). Molecular imaging of peroxynitrite with HKGreen-4 in live cells and tissues. J. Am. Chem. Soc. 136, 11728-11734.

Radi, R. (2013a). Peroxynitrite, a stealthy biological oxidant. J. Biol. Chem. 288, 26464-26472.

Radi, R. (2013b). Protein tyrosine nitration: Biochemical mechanisms and structural basis of functional effects. Acc. Chem. Res. 46, 550-559.

Redlich, S., Ribes, S., Schütze, S., Eiffert, H., and Nau, R. (2013). Toll-like receptor stimulation increases phagocytosis of Cryptococcus neoformans by microglial cells. J. Neuroinflammation 10.

Ronson, R.S., Nakamura, M., and Vinten-Johansen, J. (1999). The cardiovascular effects and implications of peroxynitrite. Cardiovasc. Res. 44, 47-59.

Schindelin, J., Arganda-Carreras, I., Frise, E., Kaynig, V., Longair, M., Pietzsch, T., Preibisch, S., Rueden, C., Saalfeld, S., Schmid, B., et al. (2012). Fiji: An open-source platform for biologicalimage analysis. Nat. Methods 9, 676-682.

Schmied, W.H., Elsässer, S.J., Uttamapinant, C., and Chin, J.W. (2014). Efficient multisite unnatural amino acid incorporation in mammalian cells via optimized pyrrolysyl tRNA synthetase/tRNA expression and engineered eRF1. J. Am. Chem. Soc. 136, 15577-15583.

Seele, J., Nau, R., Prajeeth, C.K., Stangel, M., Valentin-Weigand, P., and Seitz, M. (2016). Astrocytes enhance streptococcus suis-glial cell interaction in primary astrocyte-microglial cell co-cultures. Pathogens 5.

Shalem, O., Sanjana, N.E., and Zhang, F. (2015). High-throughput functional genomics using 
CRISPR-Cas9. Nat. Rev. Genet. 16, 299-311.

Sikora, A., Zielonka, J., Lopez, M., Joseph, J., and Kalyanaraman, B. (2009). Direct oxidation of boronates by peroxynitrite: Mechanism and implications in fluorescence imaging of peroxynitrite. Free Radic. Biol. Med. 47, 1401-1407.

Smith, M.A., Richey Harris, P.L., Sayre, L.M., Beckman, J.S., and Perry, G. (1997). Widespread peroxynitrite-mediated damage in Alzheimer's disease. J. Neurosci. 17, 2653-2657.

Sun, X., Xu, Q., Kim, G., Flower, S.E., Lowe, J.P., Yoon, J., Fossey, J.S., Qian, X., Bull, S.D., and James, T.D. (2014). A water-soluble boronate-based fluorescent probe for the selective detection of peroxynitrite and imaging in living cells. Chem. Sci. 5, 3368-3373.

Sun, X., Lacina, K., Ramsamy, E.C., Flower, S.E., Fossey, J.S., Qian, X., Anslyn, E. V., Bull, S.D., and James, T.D. (2015). Reaction-based Indicator displacement Assay (RIA) for the selective colorimetric and fluorometric detection of peroxynitrite. Chem. Sci. 6, 2963-2967.

Sun, Z.N., Wang, H.L., Liu, F.Q., Chen, Y., Tam, P.K.H., and Yang, D. (2009). BODIPY-based fluorescent probe for peroxynitrite detection and imaging in living cells. Org. Lett. 11, 18871890.

Szabó, C. (1996). The pathophysiological role of peroxynitrite in shock, inflammation, and ischemia-reperfusion injury. Shock 6, 79-88.

Szabó, C., Ischiropoulos, H., and Radi, R. (2007). Peroxynitrite: Biochemistry, pathophysiology and development of therapeutics. Nat. Rev. Drug Discov. 6, 662-680.

Tian, J., Chen, H., Zhuo, L., Xie, Y., Li, N., and Tang, B. (2011). A highly selective, cellpermeable fluorescent nanoprobe for ratiometric detection and imaging of peroxynitrite in living cells. Chem. - A Eur. J. 17, 6626-6634.

Torreilles, F., Salman-Tabcheh, S., Guérin, M.C., and Torreilles, J. (1999). Neurodegenerative disorders: The role of peroxynitrite. Brain Res. Rev. 30, 153-163.

Ueno, T., and Nagano, T. (2011). Fluorescent probes for sensing and imaging. Nat. Methods 8 , 
$642-645$.

Ueno, T., Urano, Y., Kojima, H., and Nagano, T. (2006). Mechanism-based molecular design of highly selective fluorescence probes for nitrative stress. J. Am. Chem. Soc. 128, 10640-10641.

Van Der Veen, R.C., Hinton, D.R., Incardonna, F., and Hofman, F.M. (1997). Extensive peroxynitrite activity during progressive stages of central nervous system inflammation. J. Neuroimmunol. 77, 1-7.

Weber, M., Yamada, N., Tian, X., Bull, S.D., Minoshima, M., Kikuchi, K., Mackenzie, A.B., and James, T.D. (2020). Sensing Peroxynitrite in Different Organelles of Murine RAW264.7 Macrophages With Coumarin-Based Fluorescent Probes. Front. Chem. 8.

Xia, Y., and Zweier, J.L. (1997). Superoxide and peroxynitrite generation from inducible nitric oxide synthase in macrophages. Proc. Natl. Acad. Sci. U. S. A. 94, 6954-6958.

Xiao, H., Chatterjee, A., Choi, S.H., Bajjuri, K.M., Sinha, S.C., and Schultz, P.G. (2013). Genetic incorporation of multiple unnatural amino acids into proteins in mammalian cells. Angew. Chemie - Int. Ed. 52, 14080-14083.

Xie, Z., Wei, M., Morgan, T.E., Fabrizio, P., Han, D., Finch, C.E., and Longo, V.D. (2002). Peroxynitrite Mediates Neurotoxicity of Amyloid $\beta$-Peptide 1-42- and LipopolysaccharideActivated Microglia. J. Neurosci. 22, 3484-3492.

Xu, K., Chen, H., Tian, J., Ding, B., Xie, Y., Qiang, M., and Tang, B. (2011). A near-infrared reversible fluorescent probe for peroxynitrite and imaging of redox cycles in living cells. Chem. Commun. 47, 9468-9470.

Yang, D., Wang, H.L., Sun, Z.N., Chung, N.W., and Shen, J.G. (2006). A highly selective fluorescent probe for the detection and imaging of peroxynitrite in living cells. J. Am. Chem. Soc. $128,6004-6005$.

Young, T.S., Ahmad, I., Yin, J.A., and Schultz, P.G. (2010). An Enhanced System for Unnatural Amino Acid Mutagenesis in E. coli. J. Mol. Biol. 395, 361-374. 
Yu, F., Li, P., Li, G., Zhao, G., Chu, T., and Han, K. (2011). A near-IR reversible fluorescent probe modulated by selenium for monitoring peroxynitrite and imaging in living cells. J. Am. Chem. Soc. 133, 11030-11033.

Yu, F., Li, P., Wang, B., and Han, K. (2013). Reversible near-infrared fluorescent probe introducing tellurium to mimetic glutathione peroxidase for monitoring the redox cycles between peroxynitrite and glutathione in vivo. J. Am. Chem. Soc. 135, 7674-7680.

Zhang, Q., Zhu, Z., Zheng, Y., Cheng, J., Zhang, N., Long, Y.T., Zheng, J., Qian, X., and Yang, Y. (2012). A three-channel fluorescent probe that distinguishes peroxynitrite from hypochlorite.

J. Am. Chem. Soc. 134, 18479-18482.

Zielonka, J., Sikora, A., Joseph, J., and Kalyanaraman, B. (2010). Peroxynitrite is the major species formed from different flux ratios of co-generated nitric oxide and superoxide: Direct reaction with boronate-based fluorescent probe. J. Biol. Chem. 285, 14210-14216.

Zou, M.H., Cohen, R.A., and Ullrich, V. (2004). Peroxynitrite and vascular endothelial dysfunction in diabetes mellitus. Endothel. J. Endothel. Cell Res. 11, 89-97. 
Figure 1. Peroxynitrite Biogenesis and Sensing Mechanism of pnGFP-Ultra

(A) Illustration of the major peroxynitrite $\left(\mathrm{ONOO}^{-}\right)$biogenesis pathway. $\mathrm{ONOO}^{-}$is generated from the diffusion-controlled reaction between superoxide $\left(\mathrm{O}_{2}{ }^{--}\right)$and nitric oxide $(\mathrm{NO} \bullet) . \mathrm{O}_{2}{ }^{--}$ could be produced during mitochondrial respiration (oxidative phosphorylation) or by activated NADPH oxidase (NOX). NOX can be activated during cell signaling such as a growth factor binding to a growth factor receptor (GFR). Superoxide dismutase (SOD) can convert $\mathrm{O}_{2}{ }^{--}$to hydrogen peroxide $\left(\mathrm{H}_{2} \mathrm{O}_{2}\right)$, which can mediate secondary radical (e.g. $\bullet \mathrm{OH}$ or $\left.\bullet \mathrm{OCl}\right)$ production. $\mathrm{NO} \bullet$ is produced by nitric oxide synthetase (NOS), which is coupled to arginine metabolism. $\mathrm{ONOO}^{-}$is a highly reactive nitrogen species that plays crucial roles in tyrosine nitration, lipid peroxidation, DNA damage, and cell death.

(B) Sensing mechanism of pnGFP-Ultra. pnGFP-Ultra has a boronic acid-modified chromophore that can be converted to a phenolate chromophore upon reaction with $\mathrm{ONOO}^{-}$, resulting in a large fluorescence turn-on response.

(C) Cartoon representation of pnGFP1.5-Y.Cro (PDB 5F9G) and key residues targeted during pnGFP-Ultra development. Residues identified during directed evolution that increase the brightness and folding of cpsGFP are highlighted as cyan sticks. Residues that tune of the chemoselectivity of pnGFP-Ultra are highlighted as yellow sticks. The chromophore is show in green. The inset box on the right shows the chromophore and its interacting residues. The grey sphere denotes a water molecule. The structure was prepared using Pymol.

\section{Figure 2. Sequence Alignment of pnGFP-Ultra and Related Proteins}

Mutations identified during directed evolution are highlighted in cyan. Residues subjected to rational mutagenesis are yellow-colored. The chromophore-forming residues (B denoting $p \mathrm{BoF}$ ) are highlighted in a box. Residues are numbered according to the numbering of pnGFP1.5-Y.Cro (PDB 5F9G).

\section{Figure 3. In vitro Characterization of pnGFP-Ultra}

(A) Dynamic range (expressed as $\Delta \mathrm{F} / \mathrm{F}_{0}$ ) of pnGFP-Ultra and relevant mutants towards $\mathrm{ONOO}^{-}$. $\mathrm{F}_{0}$ is the initial fluorescence intensity. $\Delta \mathrm{F}$ is the final fluorescent intensity (after treatment) minus $\mathrm{F}_{0}$. Samples were treated with $100 \mu \mathrm{M} \mathrm{ONOO}^{-}$for 1 hour.

(B) Dynamic range $\left(\Delta F / F_{0}\right)$ of pnGFP-Ultra and relevant mutants towards $\mathrm{H}_{2} \mathrm{O}_{2}$. Samples were treated with $100 \mu \mathrm{M} \mathrm{H}_{2} \mathrm{O}_{2}$ for 1 hour. 
(C) Selectivity of pnGFP-Ultra and its mutants. Arbitrarily defined selectivity is calculated as the fold of fluorescence enhancement by $\mathrm{ONOO}^{-}$/ fold of fluorescence enhancement by $\mathrm{H}_{2} \mathrm{O}_{2}$.

(D) Response of pnGFP-Ultra towards a panel of redox-active chemicals: (1) $\bullet \mathrm{OtBu}\left(1 \mathrm{mM} \mathrm{Fe}{ }^{2+}\right.$ and $100 \mu \mathrm{M} \mathrm{HOOtBu}$ ), (2) $100 \mu \mathrm{M} \mathrm{HOOtBu,} \mathrm{(3)} \bullet \mathrm{OH}\left(1 \mathrm{mM} \mathrm{Fe}^{2+}\right.$ and $\left.100 \mu \mathrm{M} \mathrm{H}_{2} \mathrm{O}_{2}\right)$, (4) 5 mM oxidized glutathione, (5) $100 \mu \mathrm{M} \mathrm{O}_{2}{ }^{--}$, (6) $100 \mu \mathrm{M}$ NOC-7 (NO•donor), (7) $100 \mu \mathrm{M} \mathrm{HOCl,}$ (8) $100 \mu \mathrm{M}$ NaHS ( $\mathrm{H}_{2} \mathrm{~S}$ donor), (9) $5 \mathrm{mM}$ L-cysteine, (10) $1 \mathrm{mM}$ DL-homocysteine, (11) $1 \mathrm{mM}$ vitamin C, (12) $100 \mu \mathrm{M} \mathrm{H}_{2} \mathrm{O}_{2}$, (13) $1 \mathrm{mM} \mathrm{H}_{2} \mathrm{O}_{2}$, (14) $100 \mu \mathrm{M} \mathrm{ONOO}^{-}$, (15) Tris buffer. (E) Linear fluorescence responses of pnGFP-Ultra $(0.2 \mu \mathrm{M})$ to $\mathrm{ONOO}^{-}$from high nanomolar to low micromolar concentrations. The limit of detection was determined to be $277 \mathrm{nM}$ with a signal-to-noise ratio $(\mathrm{S} / \mathrm{N})$ of 3 .

Figure 4. Engineering of a Highly Efficient Plasmid-based ncAAs Incorporation System in Mammalian Cells

(A) Schematics of various expression plasmids. Poly-aaRS is the poly-specific aminoacyl tRNA synthetase, eRF1-E55D is the mutant eRF1 gene, T2A is a self-cleaving 2A peptide, tRNA is the orthogonal tRNA, U6 indicates the U6 promoter, $\mathrm{H} 1$ indicates the $\mathrm{H} 1$ promoter, $\mathrm{CMV}$ is the CMV promoter, $\mathrm{EGFP}_{\mathrm{TAG}}$ is the EGFP reporter gene with a TAG codon at position 39. > or < denotes the direction of the gene cassettes.

(B) Chemical structure of $p$-Boronophenylalanine $(p \mathrm{BoF})$.

(C) Quantification of $p \mathrm{BoF}(1 \mathrm{mM})$ incorporation into the $\mathrm{EGFP}_{\mathrm{TAG}}$ reporter gene measured in a cell-based fluorescence assay. The indicated constructs were transiently expressed in HEK 293T cells and the cell lysate green fluorescence was quantified in a plate reader at $515 \mathrm{~nm}$ emission with excitation at $490 \mathrm{~nm}$. Data represent the mean \pm SD of triplicates.

\section{Figure 5. Live-cell imaging of peroxynitrite in HEK 293T cells}

(A) Constructs used for expressing pnGFP (top two plasmids) and pnGFP-Ultra (bottom two plasmids).

(B) Green fluorescent and bright-field images of cells expressing pnGFP (left) or pnGFP-Ultra (right). In the bottom row, HEK 293T cells were treated with $100 \mu \mathrm{M}$ SIN-1 for 90 min before imaging. The number of fluorescent cells from the pnGFP-Ultra-transfected group was approximately 2 -fold of that from the pnGFP-transfected group. 


\section{Figure 6. Live-cell Imaging of Peroxynitrite Production in Activated Macrophages}

(A) Mouse RAW264.7 cells were transfected with pnGFP-Ultra. After 24 hours, the samples were untreated or treated with IFN- $\gamma(50 \mathrm{U} / \mathrm{mL})$ and LPS $(100 \mathrm{ng} / \mathrm{mL})$ for 36 hours before imaging. Representative bright field (left), fluorescence (middle) and overlay (right) images of each group were shown.

(B) Quantification of fluorescence intensities of cells untreated or treated with IFN- $\gamma$ and LPS.

Gray dots indicate fluorescent intensities of single cells. Box plots represent Mean $\pm 95 \%$ confidential intervals $(\mathrm{n}=25$ individual cells for each group). Statistical test was performed using Welch's unpaired t test $(* * * *$ indicate $\mathrm{P}<0.0001)$.

\section{Figure 7. Live-cell Imaging of Amyloid $\beta$-Induced Peroxynitrite in Primary Glial Cells}

(A) Primary mouse glia cells were isolated and transfected with pnGFP-Ultra. After 24 hours, the samples were untreated or treated with amyloid $\beta(5 \mu \mathrm{M})$ for 42 hours before imaging. Representative bright field (left), fluorescence (middle) and overlay (right) images of each group were shown.

(B) Quantification of fluorescence intensities of cells untreated or treated with amyloid $\beta$. Gray dots indicate fluorescent intensities of single cells. Box plots represent Mean $\pm 95 \%$ confidential intervals ( $n=20$ and 40 individual cells for control and amyloid $\beta$-treated groups, respectively). Statistical test was performed using Welch's unpaired t test $(* * * *$ indicate $\mathrm{P}<0.0001)$. 


\section{Figure 1}

A

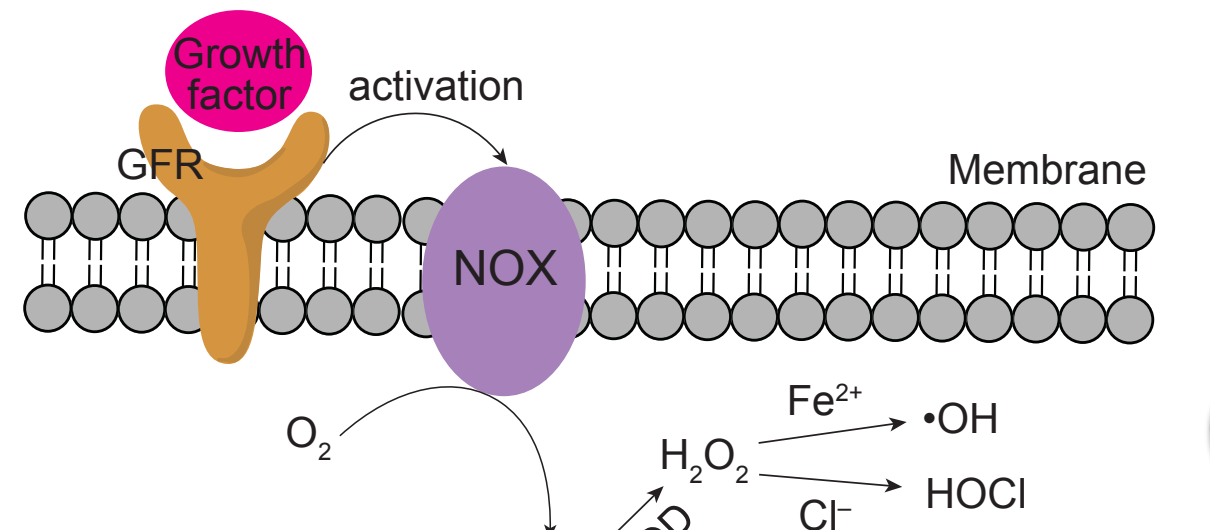

B
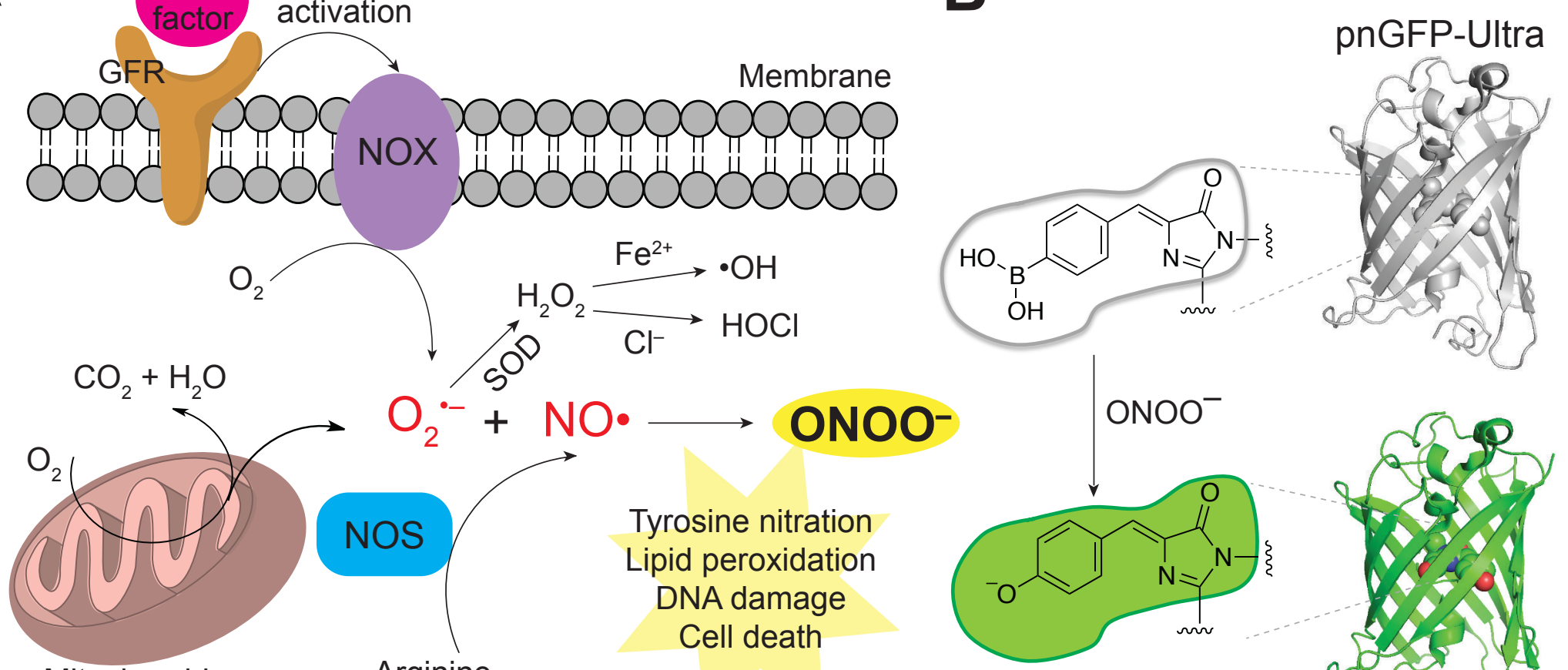

Mitochondria
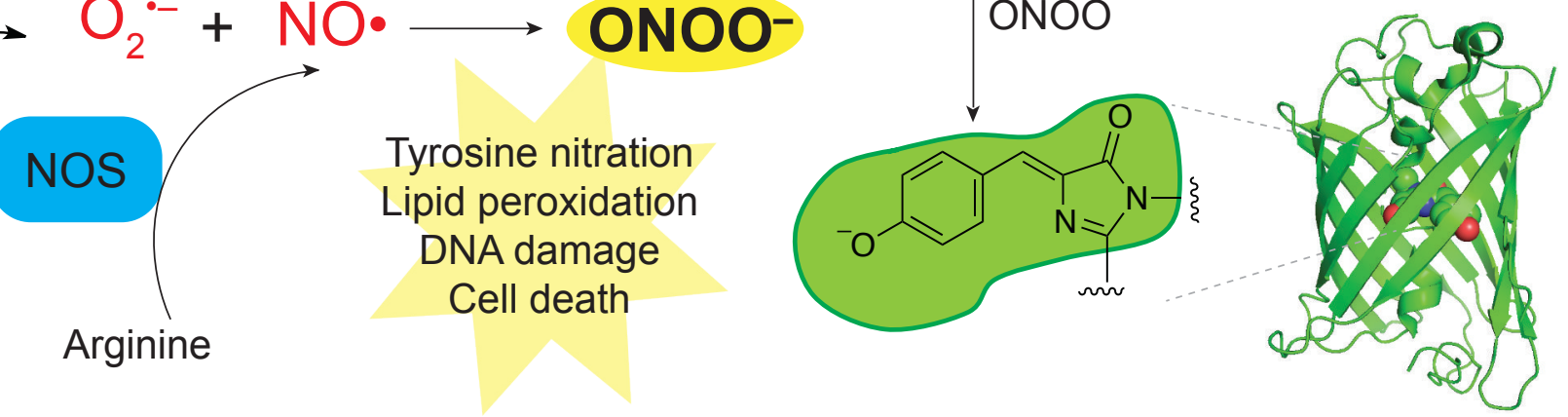

C
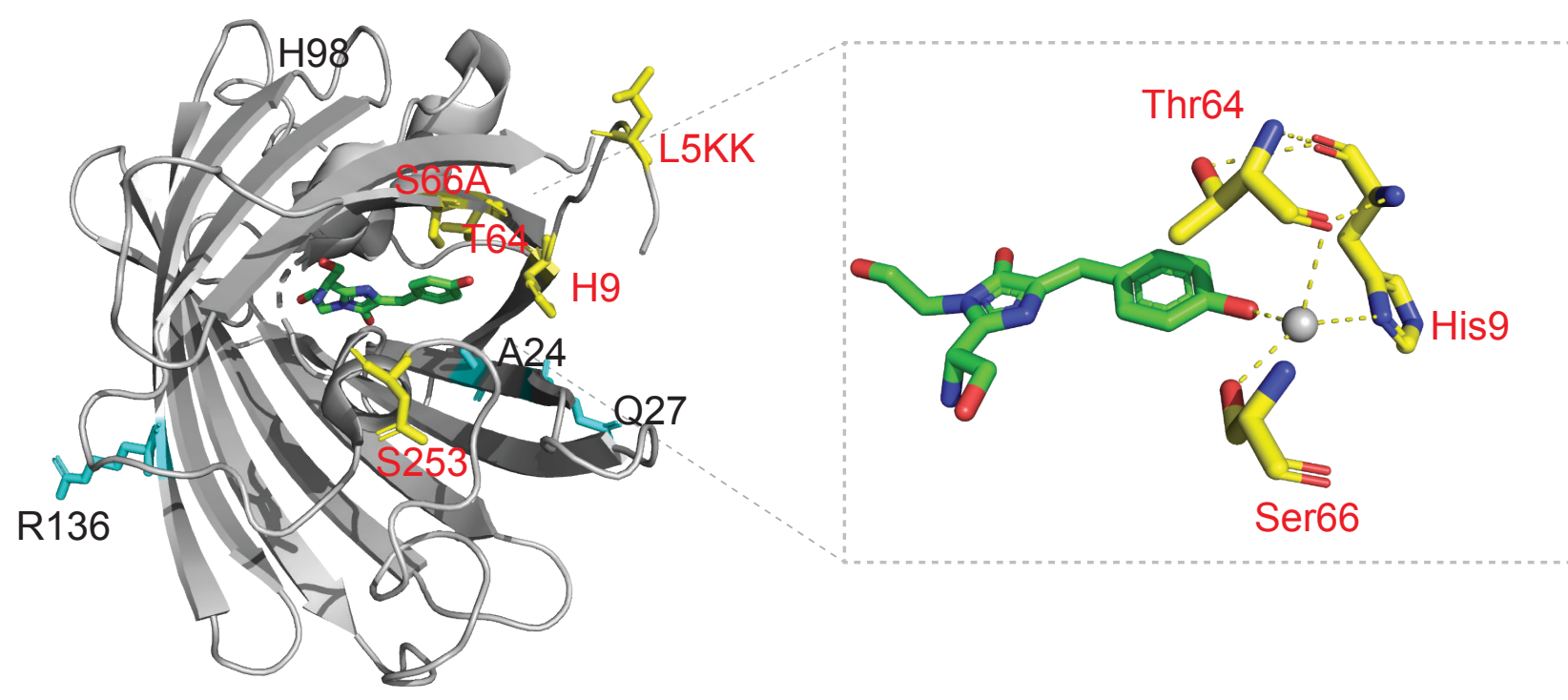


\section{Figure 2}

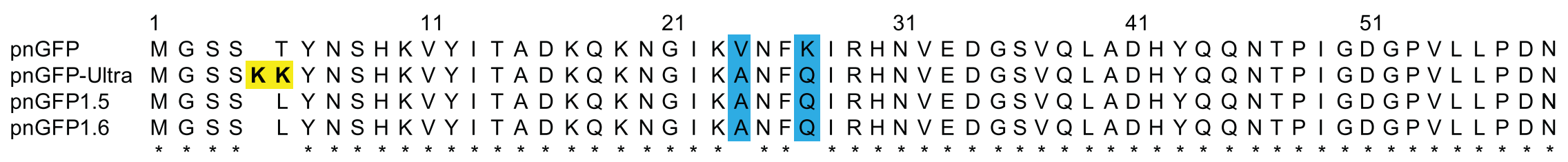
61
71
81
91
101
111

pnGFP HYLSTQSVLSKDPNEKRDHMVLLEFVTAAGITLGMDELYKVDGGSGGTGVSKGEELFTGV

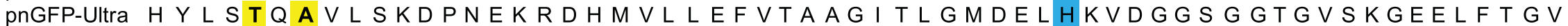
pnGFP1.5 H Y L S T Q S VL SKDPNEKRDHMVLLEFVTAAGITLGMDELHKVDGGSGGTGVSKGEELFTGV pnGFP1.6

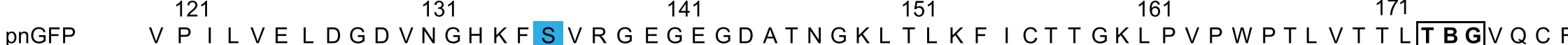
pnGFP-Ultra $\vee P I L \vee E L D G D \vee N G H K F R \vee R G E G E G D A T N G K L T L K F I C T T G K L P V P W P T L V T T L T B G V Q C F$ pnGFP1.5 $\vee P$ I L VELDGDVNGHKFRVRGEGEGDATNGKLTLKFICTTGKLPVPWPTLVT TLTBGVQCF

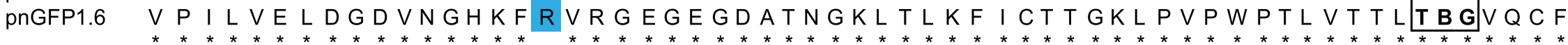
181
191
201
211
221
231

pnGFP SR Y PDHMKQHDFFKSAMPEG YVQERTIFFKDDGTYKTRAEVKFEGDTLVNRIELKGIDFK

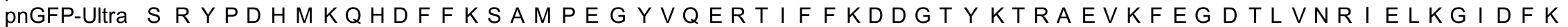
pnGFP1.5 S R Y PDHMKQHDFFKSAMPEG YVQERTIFFKDDGTYKTRAEVKFEGDTLVNR IELKGIDFK

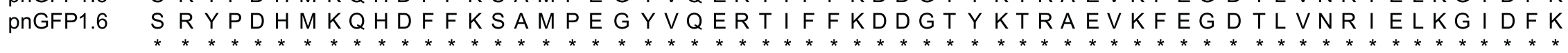

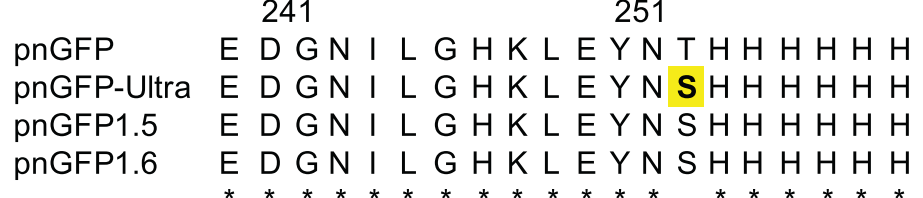




\section{Figure 3}

A B

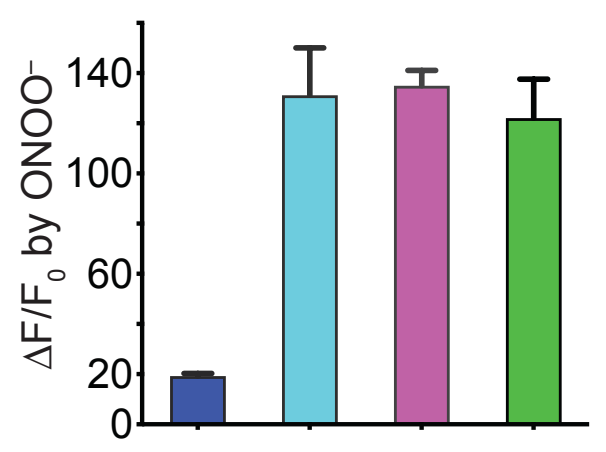

D

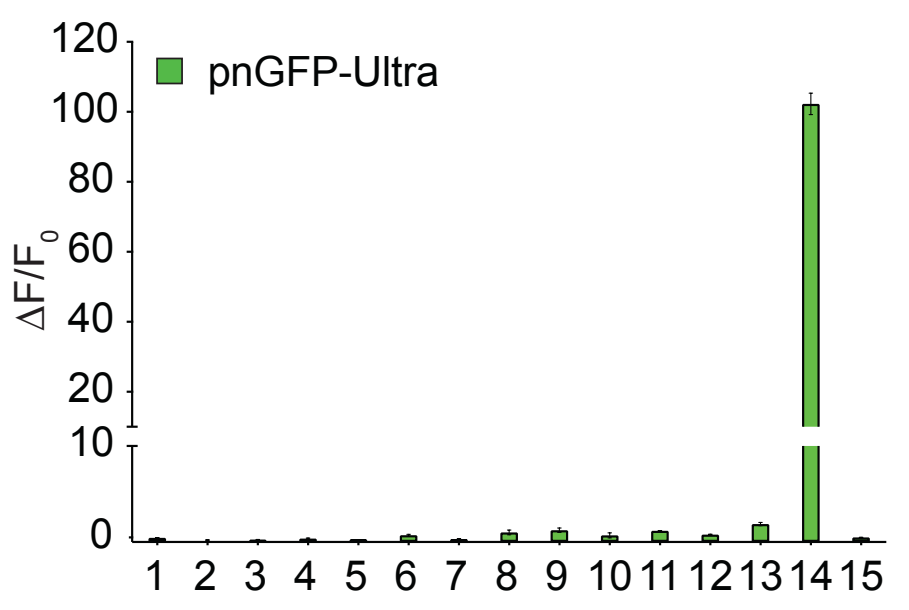

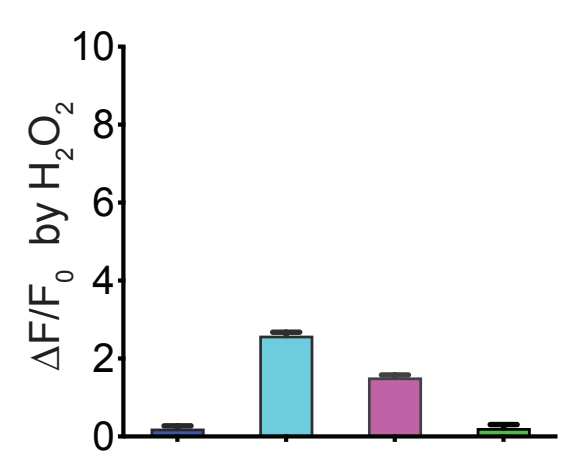

E

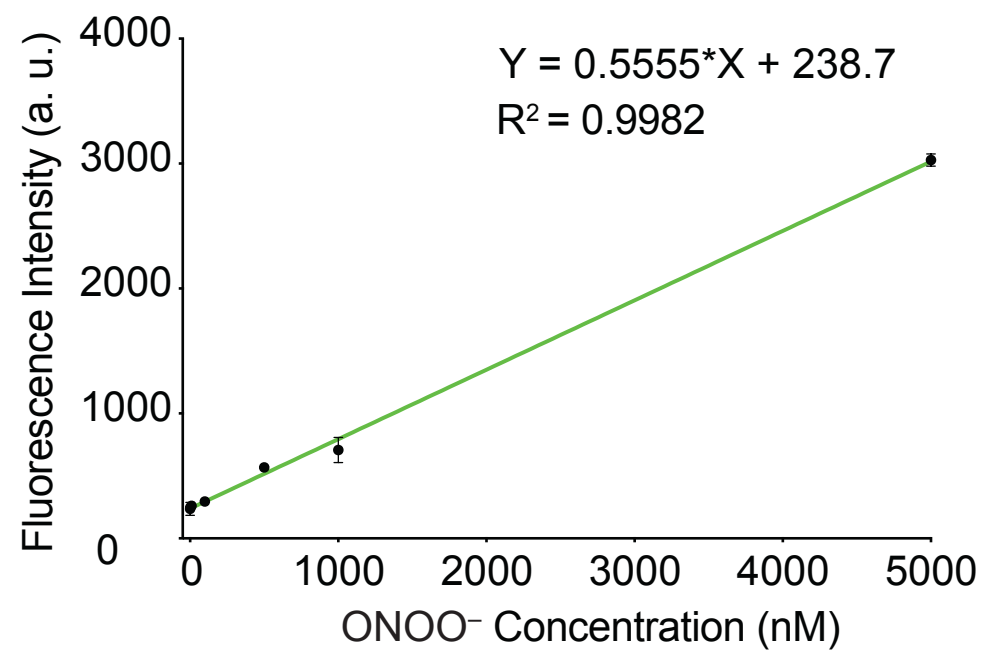

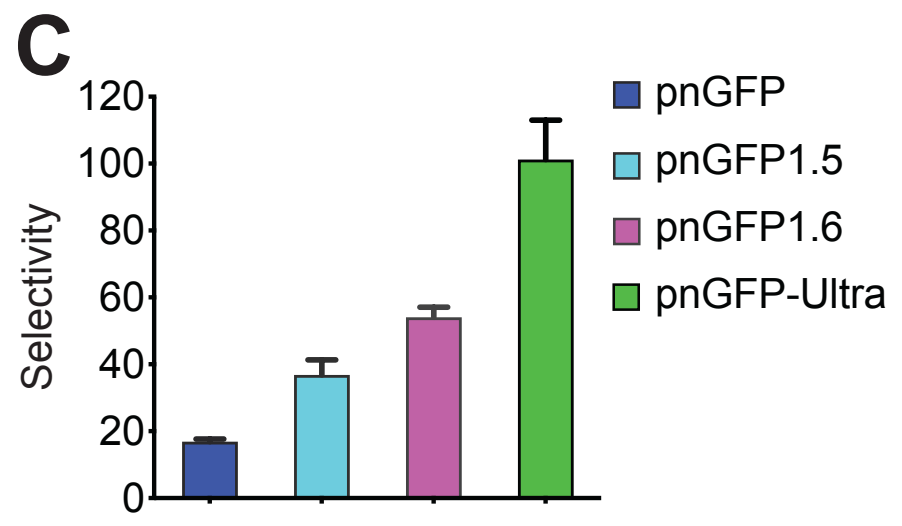

ONOO- Concentration (nM) 
Figure 4

A $a-C M V>-E_{E G P P}$

$b-$ tRNA $<$ H1-tRNA $<U 6$-tRNA $<$ H1-tRNA $<U 6-C M V>-$ Poly-aaRS

$c-\operatorname{tRNA}<\mathrm{H} 1-\operatorname{tRNA}<\mathrm{U} 6-\operatorname{tRNA}<\mathrm{H1}-\mathrm{tRNA}<\mathrm{U} 6-\mathrm{CMV}>-\mathrm{EGFP}_{\mathrm{TAC}}$

$d-$ tRNA $<$ H1-tRNA $<$ U6-tRNA $<$ H1-tRNA $<$ U6- CMV > Poly-aaRS T2A eRF1 E55D

e - tRNA $<$ H1-tRNA $<$ U6-tRNA $<$ H1-tRNA $<$ U6- CMV > EGFP TAG $_{-}-\mathrm{CMV}>-$ Poly-aaRS -

B

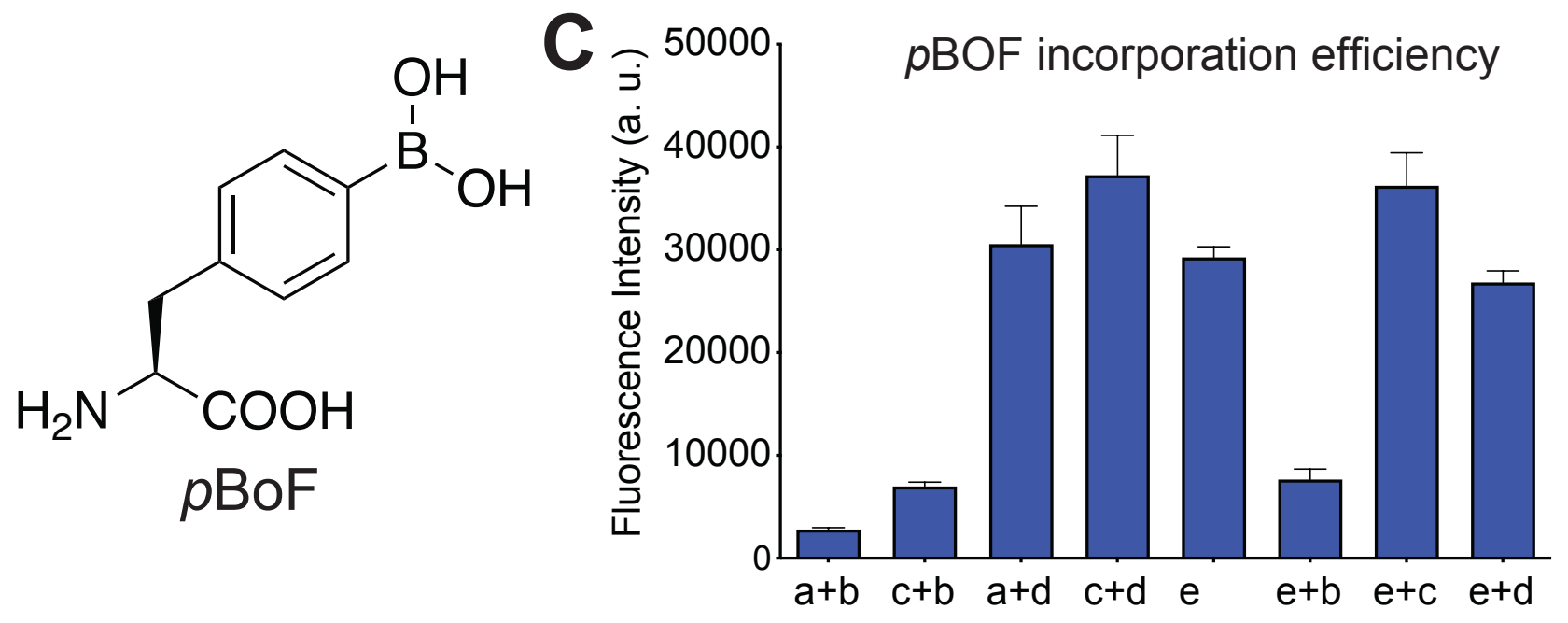


Figure 5

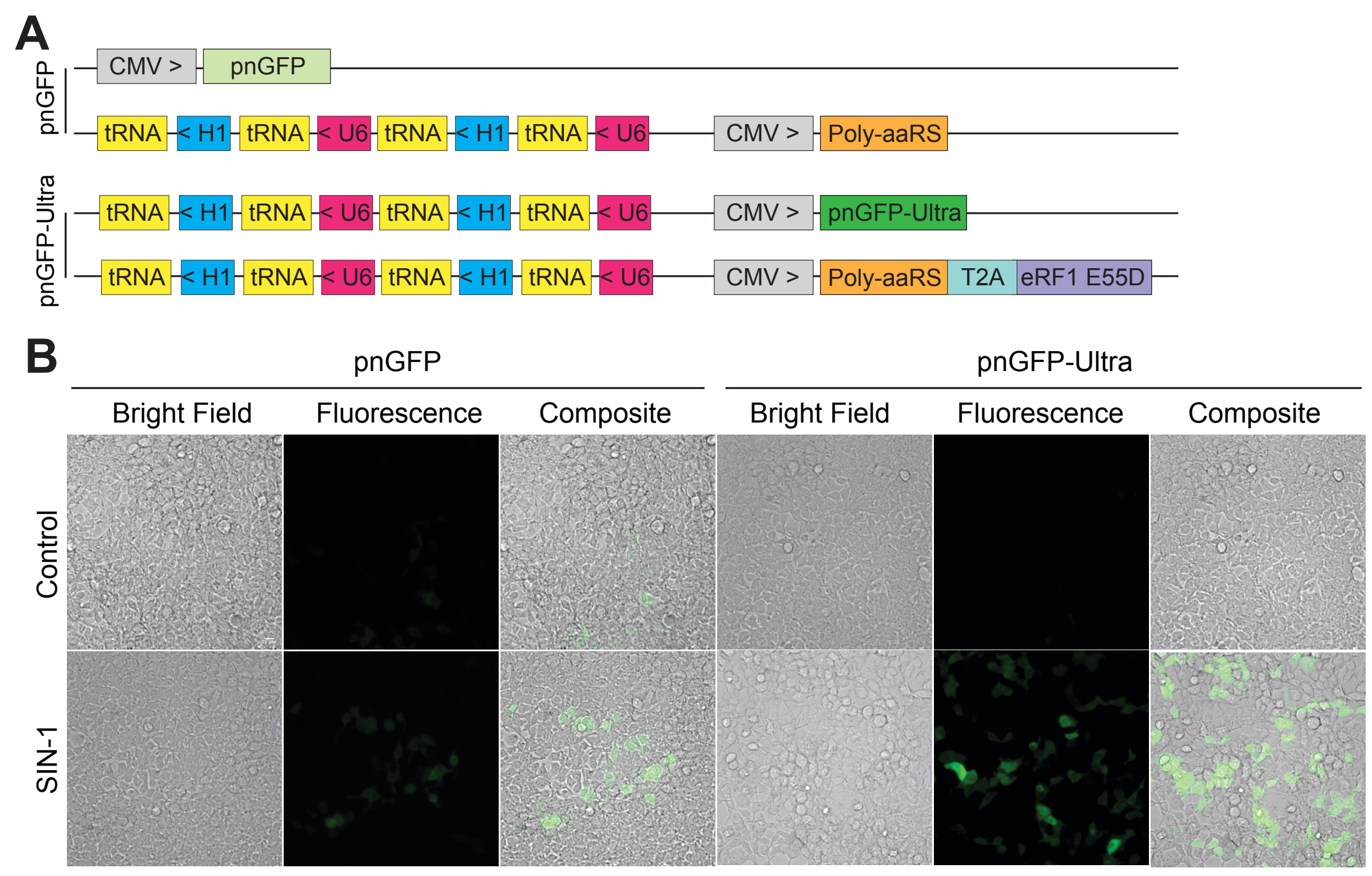


Figure 6

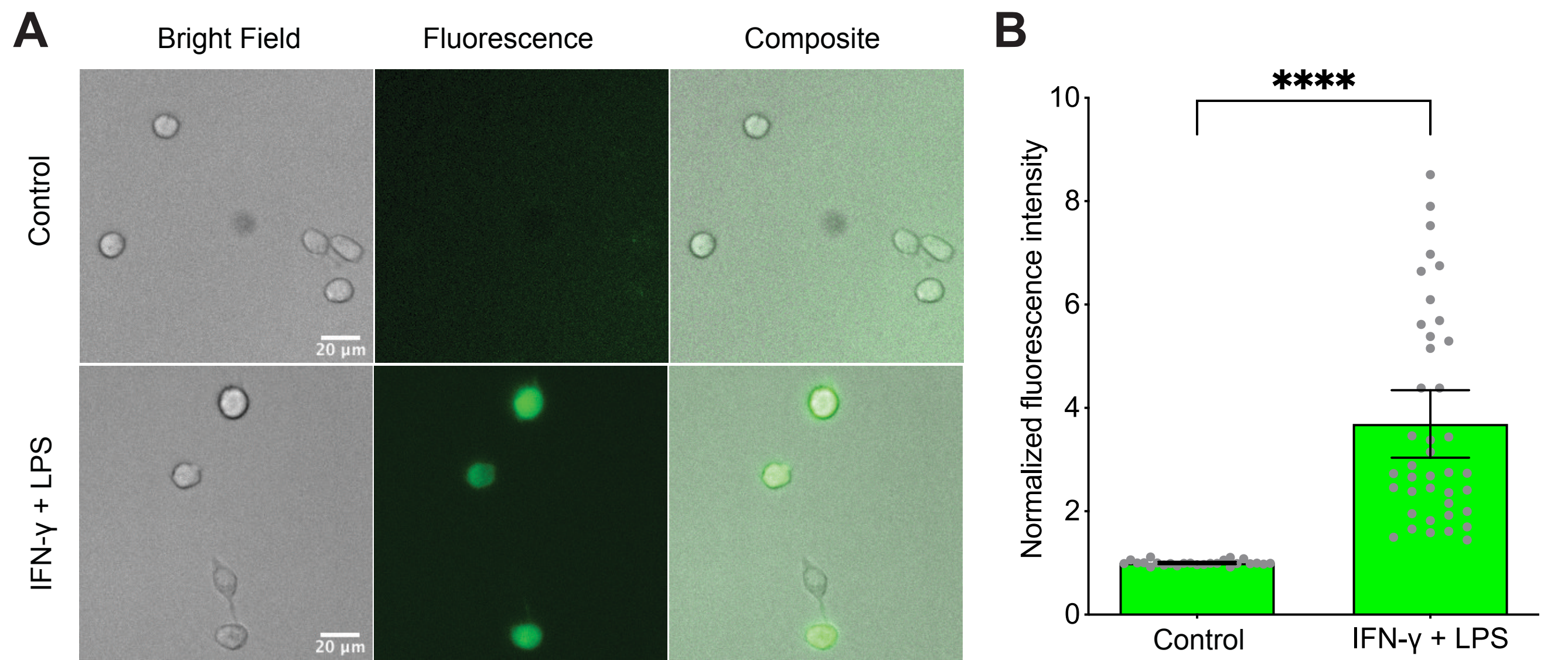


Figure 7

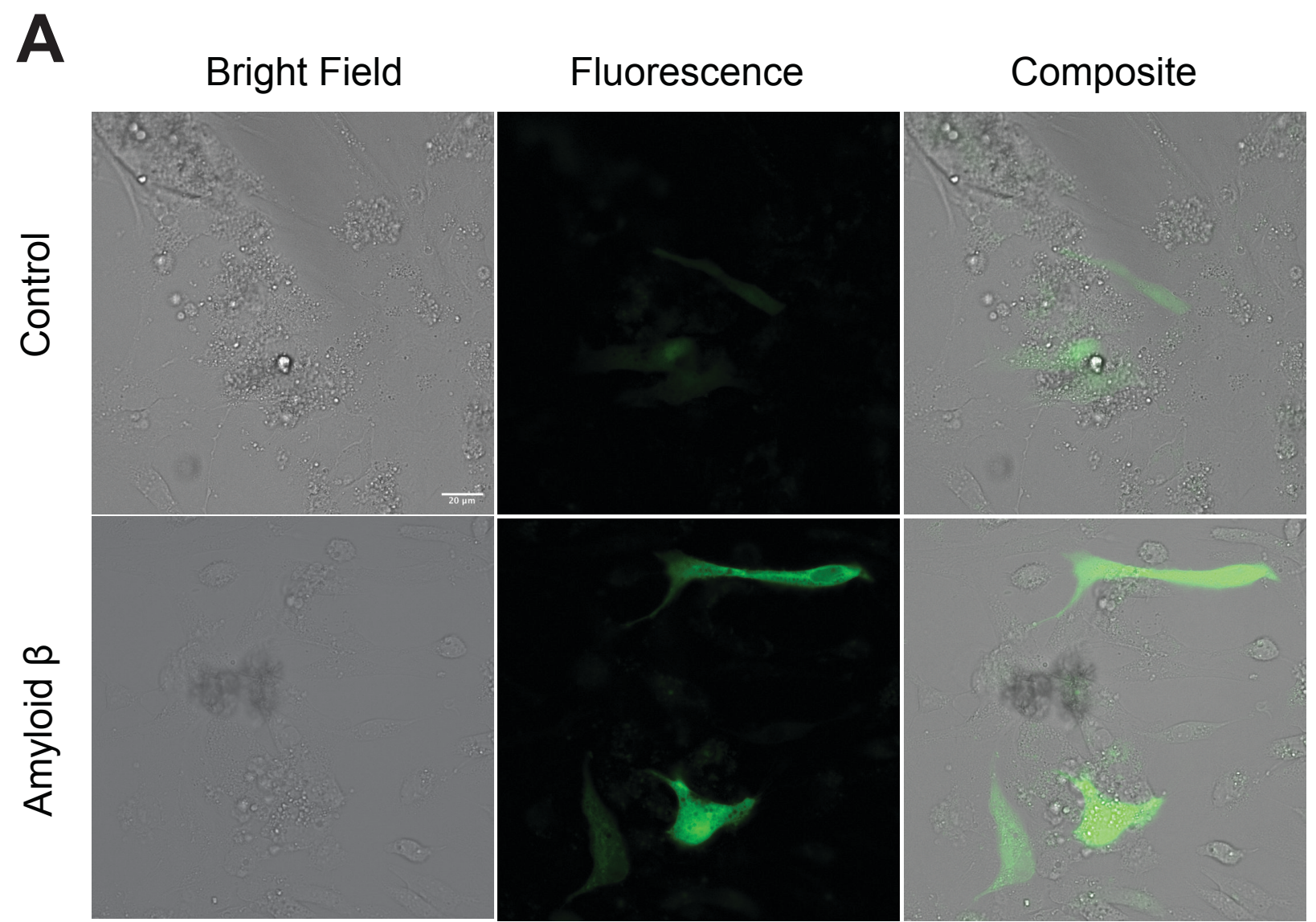

B

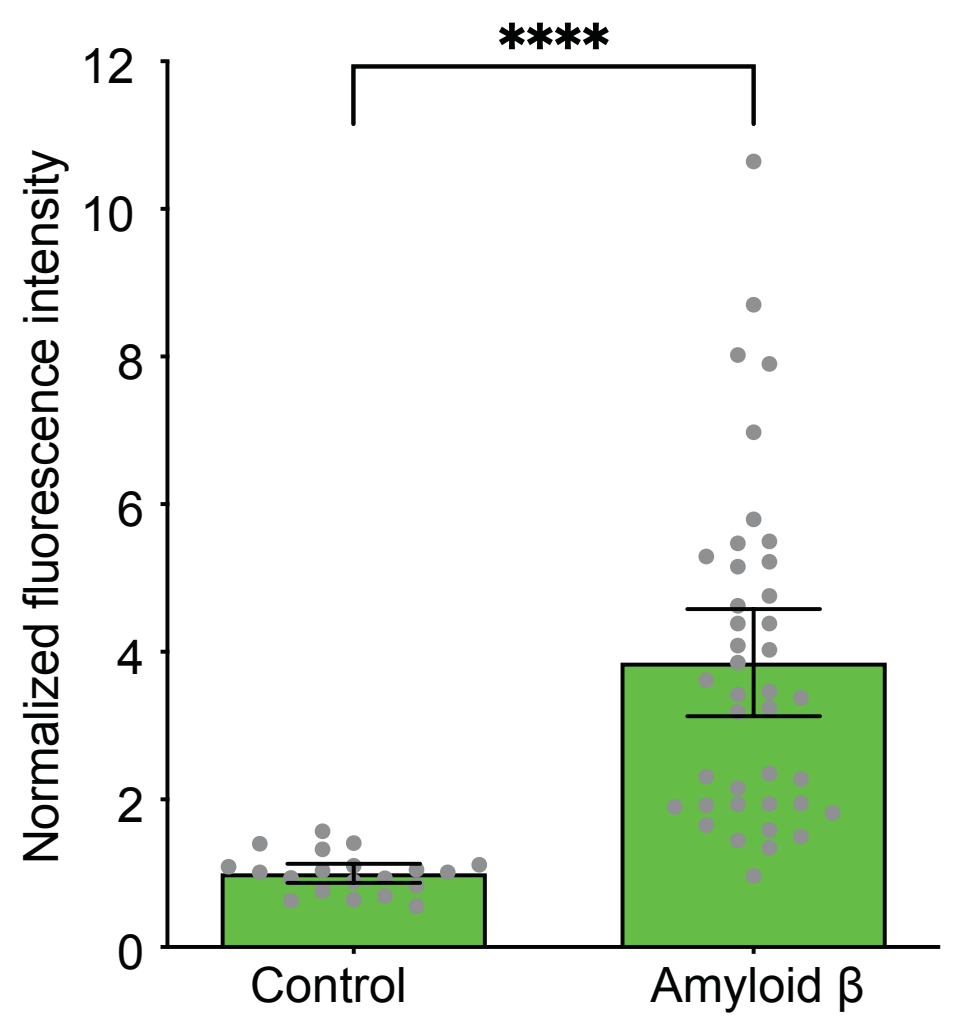

